\title{
Motor protein-dependent transport of AMPA \\ receptors into spines during long-term potentiation
}

Susana S. Correia ${ }^{1}$, Silvia Bassani ${ }^{3}$, Tyler C. Brown ${ }^{1,2}$, Marie-France Lisé ${ }^{4}$, Donald S. Backos ${ }^{1}$, Alaa El-Husseini ${ }^{4,5}$, Maria Passafaro ${ }^{3}$ and José A. Esteban $^{1,2, *}$

${ }^{1}$ Department of Pharmacology and ${ }^{2}$ Neuroscience Program, University of Michigan Medical School, 1150 W. Medical Center Dr., Ann Arbor, MI 48109-0632, USA

${ }^{3}$ DTI Dulbecco Telethon Institute, CNR Institute of Neuroscience, Cellular and Molecular Pharmacology, Department of Pharmacology, University of Milan, Via Vanvitelli 32, 20129 Milano, Italy

${ }^{4}$ Department of Psychiatry, Brain Research Centre, Faculty of Medicine, University of British Columbia, 2255 Wesbrook Mall, Vancouver, BC, V6T 1 Z3 Canada

${ }^{5}$ We wish to dedicate this work to the memory of Alaa El-Husseini, a great friend and an outstanding scientist.

*Corresponding author: Dept. of Pharmacology, Univ. of Michigan Medical School, 1150 W. Medical Center Dr., Ann Arbor, MI 48109-0632, FAX: 734-763-4450; e-mail: estebani@umich.edu

Running title: Myosin Va-dependent transport of AMPA receptors 


\section{SUMMARY}

The regulated trafficking of neurotransmitter receptors at synapses is critical for synaptic plasticity. Still, the molecular machinery that controls active transport of receptors into synapses is largely unknown. We report that, in rat hippocampus, the insertion of AMPA receptors into spines during synaptic plasticity requires a specific motor protein, which we identify as Myosin Va. We found that Myosin Va associates with AMPA receptors through its cargo binding domain. This interaction is enhanced by active, GTP-bound Rab11, which is also transported by the motor protein. Myosin Va mediates the CaMKIItriggered translocation of GluR1 receptors from the dendritic shaft into spines, but it is not required for constitutive GluR2 trafficking. Accordingly, Myosin Va is specifically required for long-term potentiation, but not for basal synaptic transmission. In summary, this work identifies the specific motor protein and organelle acceptor that catalyze the directional transport of AMPA receptors into spines during activity-dependent synaptic plasticity. 


\section{INTRODUCTION}

The polarized trafficking and targeting of a variety of proteins, such as neurotransmitter receptors, ion channels, anchoring and cell adhesion molecules are critically important for synaptic function and plasticity. In particular, local membrane transport at the postsynaptic terminal is now appreciated as an important contributor to synaptic plasticity (see ${ }^{1}$ for a recent review). In the case of excitatory synapses in the hippocampus, the postsynaptic membrane is located within micron-size dendritic protrusions, known as spines. Despite the small distances involved, the transport of specific proteins and organelles in and out of spines appears to be tightly regulated. For example, synaptic potentiation is accompanied by the translocation of recycling endosomes $^{2}$ and AMPA-type glutamate receptors (AMPARs) into spines ${ }^{3}$. Conversely, the A-type $\mathrm{K}^{+}$channel $\mathrm{Kv4.2}$ is mobilized in the opposite direction, that is, leaves the spine, upon similar synaptic plasticity induction ${ }^{4}$. This dynamic behavior implies a carefully choreographed transport of specific cargo in a polarized manner in response to synaptic activity. Nevertheless, the molecular mechanisms that control this directional trafficking in dendritic spines remain to be discovered.

AMPARs are responsible for most excitatory synaptic transmission in the brain, and their regulated addition and removal from synapses leads to long-lasting forms of synaptic plasticity, such as long-term potentiation (LTP) and long-term depression $(\mathrm{LTD})^{5}$. Distinct AMPAR populations undergo differential synaptic trafficking depending on their subunit composition. Thus, AMPARs assembled as GluR2 homomers or GluR2/GluR3 heteromers cycle in and out of the synaptic membrane in a constitutive manner, which does not require synaptic activity ${ }^{6,7}$. This constitutive trafficking requires 
GluR2-specific interactions with NSF ${ }^{8-10}$. In contrast, AMPARs containing the GluR1 subunit translocate into spines and are inserted into synapses in response to NMDA receptor activation during $\mathrm{LTP}^{3,11}$.. However, the molecular machinery that mediates the acute, unidirectional transport of GluR1 receptors into spines and synapses during LTP is unknown.

F-actin is the predominant cytoskeletal element in dendritic spines. Therefore, actin filaments may serve as transport tracks for the delivery or removal of proteins at postsynaptic terminals. However, to this date, the actin cytoskeleton has been commonly associated with morphogenesis and structural dynamics of spines (see ${ }^{12}$ for a recent review), but not with synaptic trafficking. The active transport of cargo along actin filaments is carried out by molecular motor proteins of the myosin family ${ }^{13}$. Therefore, myosin motors are attractive candidates to mediate directional trafficking of synaptic proteins. Some members of the myosin family have been shown to participate in AMPAR transport, such as Myosin VI for endocytosis ${ }^{14}$ and Myosin Vb for dendritic surface expression ${ }^{15}$. However, it remains to be determined whether a specific motor protein is involved in the synaptic delivery of AMPARs. In fact, it has been described that AMPARs can reach their synaptic targets by passive diffusion along the extrasynaptic dendritic surface ${ }^{16}$, potentially arguing against the requirement for an active transport process during synaptic delivery. Nevertheless, the existence of constitutive and activity-dependent synaptic delivery of AMPARs ${ }^{7}$ leaves open the possibility that these trafficking modes may have different requirements for active protein transport. 
Myosin $\mathrm{Va}$ is highly expressed in brain $^{17}$ and is present in the postsynaptic density $(P S D)^{18,19}$ as well as in light membrane fractions ${ }^{20}$. In humans, mutations in Myosin Va are associated with Griscelli $^{21}$ and Elejalde ${ }^{22}$ syndromes, which are characterized by severe neurological impairments (seizures, mental retardation and hypotonia). In mouse, the spontaneous null mutation of Myosin Va (dilute-lethal) also causes severe neurological abnormalities that culminate in death 2 to 3 weeks postnatal ${ }^{17}$. These pathologies suggest that Myosin Va is involved in critical intracellular transport events required for proper neuronal function and cognition.

Myosin $\mathrm{Va}$ is involved in the transport of membrane organelles in axons ${ }^{23}$, but little is known about its potential function in postsynaptic membrane trafficking. In the cerebellum of dilute-lethal mice, the spines of Purkinje cells lack smooth endoplasmic reticulum and inositol-1,4,5-trisphosphate receptors (IP3Rs) ${ }^{24,25}$, and LTD of parallel fiber synapses is abolished ${ }^{26}$. It has been previously published that synaptic function and plasticity at CA3-CA1 hippocampal synapses is preserved in the dilute-lethal mice $^{27}$. However, there have been reports of multiple synaptic dysfunctions in other Myosin Va mutant mice ${ }^{28}$. In addition, more acute disruption of Myosin Va function with a dominant negative construct impairs the transport of mRNA/protein complexes into dendritic spines in hippocampal neurons ${ }^{20}$, suggesting that Myosin Va may play a role in synaptic trafficking in the hippocampus.

In this study we have explored the role of Myosin Va in AMPAR trafficking and synaptic plasticity in CA1 hippocampal neurons. We have found that Myosin Va associates with AMPARs through its globular, cargo-binding domain. Furthermore, this association is enhanced by the vesicular protein Rab11. Using a combination of 
molecular biology, electrophysiology, and fluorescence imaging, we show that Myosin Va mediates a very distinct step in AMPAR trafficking, that is, the activity-dependent translocation of GluR1-containing receptors from the dendritic shaft into the spine compartment. Therefore, our data reveal a specialized role of Myosin Va in postsynaptic function, by mediating short-range vesicular transport into spines during synaptic plasticity. 


\section{RESULTS}

\section{Association between AMPARs, Myosin Va and Rab11 in neurons}

In order to identify novel interacting proteins that may regulate AMPAR function, we carried out a preparative immunopurification from hippocampal neuronal cultures. AMPARs were immunopurified with GluR2 C-terminal antibodies. Since most AMPARs in the hippocampus are hetero-oligomers composed of GluR1/GluR2 or GluR2/GluR3 subunits $^{29}$, this approach is expected to yield AMPAR-associated proteins irrespectively from receptor subunit composition. The presence of co-purified proteins was evaluated by SDS-gel electrophoresis and silver staining (Fig. 1a). Mass spectroscopy analysis of the high-molecular weight bands identified Myosin $\mathrm{Va}$ as one of the coimmunoprecipitated proteins (M.W. $\sim 217 \mathrm{KDa}$ ). The association between AMPARs and Myosin Va was confirmed using standard immunoprecipitations with antibodies against GluR1 and GluR2, and Western blot analysis (Fig. 1b). Interestingly, the small GTPase associated to recycling endosomes, Rab11 ${ }^{30}$, was also found to co-precipitate with AMPARs. As expected, the GluR2 PDZ binding partner GRIP1 was co-precipitated, whereas the NMDAR subunit NR1 did not associate with Myosin Va or AMPARs under these conditions (Fig. 1b).

As an independent method to test the interaction between AMPARs and Myosin Va, we carried out GST-pull downs with different domains of the Myosin Va C-terminus. As shown in Fig. 1c (left panels), the globular tail of Myosin Va ("G-tail"; cargo binding domain $^{31}$ ) interacts with AMPAR subunits from hippocampal neurons, whereas the medial tail of Myosin V ("M-tail", coiled-coil domain) or plain GST did not. In addition, pull-downs carried out with recombinant proteins expressed in COS-7 cells, indicated 
that the globular tail of Myosin Va interacts with Rab11 and with full-length GluR1, but not with a truncated GluR1 mutant lacking the last 30 amino acids of the C-terminus (GluR1- $430 ;$ Fig. 1c, middle panels). And finally, His-tag-purified GluR1 C-terminus (last 50 amino acids) was also pulled-down by the globular tail of Myosin Va (Fig. 1c, right panels), strongly suggesting that there is a direct interaction between these two proteins. In conclusion, these combined data indicate that AMPARs and Myosin Va associate in neurons, and that this complex is mediated by a direct interaction between the cargo binding domain of Myosin Va and the C-terminal 30 amino acids of GluR1.

To further evaluate the association between AMPARs and Myosin Va, we carried out immunolabeling analyses of the endogenous proteins in dendrites from hippocampal neurons. Myosin Va shows a partial co-localization with GluR1 and GluR2 subunits in primary neuronal cultures (Fig. 2a,b). In many instances, though, Myosin Va appeared to localize adjacent to AMPAR puncta (see high-magnification insets in lower panels). We also examined the subcellular distribution of endogenous Myosin Va in dendrites and spines from CA1 neurons in hippocampal slices. Myosin Va was mostly localized at dendritic shafts, as compared with the enrichment of actin and PSD95 at spines (Fig. 2c,d; see high-magnification insets in right panels). These observations are consistent with the previously reported partial co-localization between Myosin Va and PSD95 in primary neuronal cultures ${ }^{19}$.

\section{Interference with Myosin Va depresses synaptic responses}

In order to start evaluating a potential role of Myosin Va in AMPAR trafficking and/or synaptic plasticity, we overexpressed the cargo binding domain (globular tail) of 
Myosin Va in CA1 hippocampal neurons. This domain displaces endogenous Myosin Va from its tail-associated $\operatorname{cargo}^{31}$, therefore acting as a dominant negative construct (Myosin Va-dn). This experimental strategy has been employed on multiple occasions to interfere with Myosin Va-dependent trafficking. Importantly, this approach is specific enough to distinguish distinct functions of the three different Myosin $\mathrm{V}$ isoforms: $\mathrm{Va}, \mathrm{Vb}$ and $\mathrm{Vc}^{15,32,33}$.

We expressed GFP-fusion proteins of Myosin Va-dn, Myosin Vb-dn or Myosin VIdn in CA1 hippocampal neurons from organotypic slice cultures, as described in Methods. The effect of these dominant negative proteins on synaptic transmission was evaluated by simultaneous double whole-cell recordings form pairs of nearby infected and uninfected neurons, under voltage clamp. Myosin Va-dn produced a small but significant depression of AMPAR- and NMDAR-mediated currents, as compared to uninfected cells, while $G A B A_{A}$ receptor-mediated inhibitory currents were unchanged (Fig. 3a-c; left-most panels). Neither Myosin VI-dn (Fig. 3a, middle panels) nor Myosin Vb-dn (Supplementary Fig. 1) significantly affected AMPAR-mediated transmission. To note, Myosin Va-dn did not affect the expression levels nor phosphorylation stage of multiple synapse-associated proteins (Supplementary Fig. 2). Therefore, these results suggest that Myosin Va plays a role in AMPAR synaptic function, which is not shared by other myosin isoforms.

Hippocampal slices in culture exhibit spontaneous activity, which can drive AMPARs into synapses ${ }^{34}$. To determine whether the depression of AMPAR responses induced by Myosin Va-dn is dependent on spontaneous activity, we performed double whole-cell recordings from slices in which spontaneous activity was blocked with high 
$\mathrm{Mg}^{2+}(12 \mathrm{mM} \mathrm{MgCl}$ ) or the sodium channel blocker tetrodotoxin $(1 \mu \mathrm{M})$ during the expression of the recombinant protein $^{34}$ (slices are returned to the standard solution during the recordings; see Methods). Under these conditions Myosin Va-dn failed to depress AMPAR or NMDAR transmission (Fig. 3a,b; two right-most panels), suggesting that Myosin Va plays a specific role in activity-dependent regulation of synaptic function. Interestingly, these results also suggest that there is an activity- dependent co-scaling between AMPA and NMDA receptor-mediated synaptic transmission, as it has been previously observed in response to spontaneous synaptic activity ${ }^{35}$..

Although these dominant negative constructs were expressed in postsynaptic CA1 neurons, we wanted to examine whether Myosin Va-dn may be retrogradely interfering with presynaptic mechanisms. To this end, we measured paired pulse facilitation (PPF), as an indicator of presynaptic function, from Myosin Va-dn-infected and uninfected CA1 neurons in hippocampal slices. As shown in Fig. 3d, PPF was unaltered by Myosin Vadn expression. These results confirm that the depression of synaptic transmission observed with Myosin Va-dn is not due to alteration of presynaptic properties, and suggest that Myosin Va plays an independent postsynaptic role at excitatory hippocampal synapses.

\section{Myosin Va is required for LTP and GluR1 synaptic delivery}

AMPARs continuously cycle in and out of synapses in an NSF-dependent manner ${ }^{9,10}$. Our observation that Myosin Va-dn does not affect basal transmission in the absence of spontaneous neuronal activity suggests that Myosin $\mathrm{Va}$ is not involved in this constitutive trafficking. In order to directly test this interpretation, we carried out 
intracellular infusion experiments with a short peptide that interferes with the GluR2NSF interaction. This peptide has been shown to produce a fast "run-down" of synaptic transmission ${ }^{9,10}$, as AMPARs are continuously internalized but fail to be reinserted at the synaptic membrane. Neurons expressing Myosin Va-dn displayed virtually identical "run-down" of synaptic transmission as uninfected neurons (Fig. 4a,b). As control, a peptide that does not bind NSF, pep4c ${ }^{9}$, did not produce "run-down" of transmission (Fig. 4b). Therefore, these results confirm our interpretation that Myosin $\mathrm{Va}$ is not required for the constitutive synaptic trafficking of AMPARs.

In order to investigate more directly whether Myosin $\mathrm{Va}$ is involved in the activitydependent delivery of AMPARs into synapses, we evaluated the effect of Myosin Va-dn in long term potentiation (LTP) in CA1 hippocampal neurons. Hippocampal slices were infected with Myosin Va-dn and were incubated with high $\mathrm{Mg}^{2+}$ to block spontaneous activity during the expression time. Importantly, NMDA and AMPA receptor currents are not affected by the recombinant protein under these conditions (Fig. 3a,b, right-most panels), and therefore, we can directly evaluate the effect of Myosin Va-dn in LTP expression without interfering with LTP induction. After $14 \mathrm{~h}$, slices were transferred to normal extracellular solution (see Methods) and LTP was induced on infected and uninfected CA1 neurons by pairing presynaptic stimulation $(3 \mathrm{~Hz}, 1.5$ min) with postsynaptic depolarization ( $0 \mathrm{mV}$; see Methods). As shown in Fig. 4c,d, uninfected neurons showed a robust, 2.5 -fold potentiation of transmission. In contrast, LTP was virtually abolished in Myosin Va-dn-expressing neurons. To note, Myosin Va-dn did not have any effect on the non-potentiated (control) pathway (Fig. 4d). 
As an alternative method to test the role of Myosin Va in LTP, we used an RNA interference approach. A small interference RNA (siRNA) was designed against the rat Myosin Va mRNA (see Methods for construct details). This siRNA was shown to effectively down-regulate the expression of recombinant Myosin Va in transfected COS7 cells, and of endogenous Myosin Va in hippocampal neurons (Supplementary Fig. 3). To test the role of endogenous Myosin Va in LTP, we expressed the anti-Myosin Va siRNA in CA1 hippocampal neurons for three days while blocking spontaneous activity with tetrodotoxin. Slices were then transferred to regular recording solution and LTP was induced as described above. As shown in Fig. 4c,d, knock-down of Myosin Va with siRNA virtually abolished synaptic potentiation. Therefore, these combined results using Myosin Va-dn and siRNA strongly suggest that Myosin Va is critically required for LTP.

In the hippocampus, expression of LTP is mediated by synaptic addition of GluR1containing AMPA receptors into synapses ${ }^{11}$. To specifically investigate the role of Myosin Va on the synaptic delivery of GluR1 AMPA receptors, we used the biolistic system to co-transfect CA1 hippocampal neurons with GFP-GluR1 and constitutively active CamKII (tCamKII), previously shown to mimic LTP and drive GluR1 to synapses $^{11}$. Delivery of GFP-GluR1 receptors to synapses is monitored using the inward rectification properties of the homomeric recombinant receptor (electrophysiological tagging ${ }^{11,36}$ ). Synaptic delivery is then quantified as an increase in the ratio of the evoked postsynaptic current at $-60 \mathrm{mV}$ relative to the current at $+40 \mathrm{mV}$ (rectification index, $\mathrm{RI}=\mathrm{I}_{-60} / \mathrm{I}_{+40}$ ). Co-expression of GluR1 and tCamKII produced a significant increase in the rectification index as compared to untransfected cells (Fig. 4e; see also ${ }^{11}$ ). In contrast, co-expression of Myosin Va-dn together with GluR1 and 
tCamKII completely blocked the increase in rectification (Fig. 4e). Similar results were obtained by knocking-down Myosin Va with RNA interference, that is, anti-Myosin Va siRNA abolished the increase in rectification while a control scrambled siRNA did not

(Fig. 4f). These results strongly suggest that synaptic delivery of GluR1 induced by CamKII is mediated by Myosin Va.

PSD95 overexpression can also drive the insertion of GluR1 into hippocampal synapses $^{37}$. Therefore, we tested whether PSD95-driven delivery of GluR1 requires Myosin Va function. As expected, co-transfection of GluR1 with PSD95 lead to an increase of the rectification index (Supplementary Fig. 4a), indicating delivery of recombinant GluR1 into synapses (see also ${ }^{37}$ ). Interestingly, Myosin Va-dn also blocked this delivery (Supplementary Fig. 4b).

In conclusion, these combined data using dominant negative and RNA interference approaches indicate that Myosin Va is required for all tested forms of regulated synaptic delivery of GluR1: LTP induction, CaMKII activation and PSD95 overexpression. Therefore, Myosin Va appears to be a central factor for the activity-dependent transport of AMPARs into synapses.

\section{Myosin Va is not required for dendritic AMPAR transport}

The presence of Myosin $\mathrm{Va}$ at spines and the PSD raises the possibility that Myosin Va transports AMPARs locally at synapses. However, we have found that Myosin Va is mostly localized in dendrite shafts (Fig. 2c,d), where it could transport organelles involved in the dendritic trafficking of AMPARs. To determine the specific step mediated by Myosin Va in the transport of AMPARs, we co-transfected organotypic 
hippocampal slices with RFP-tagged Myosin Va-dn, Myosin Vb-dn or plain RFP (as control) together with GFP-tagged AMPAR subunits (tCaMKII was also co-expressed with GFP-GluR1 in some of the cells, as indicated) (Fig. 5a,d). The efficiency of the dendritic transport of AMPARs was quantified from the fluorescence intensity of the GFP-tagged receptor along the primary apical dendrite, and plotted as a function of the distance from the cell body ${ }^{36}$. GFP fluorescence is normalized to the maximum value at the cell body, to control for variability in expression levels.

As shown in Fig. 5b (GluR1) and e (GluR2), neither Myosin Va- nor Myosin Vb-dn affected the distribution of GFP-tagged receptors along dendrites. Specifically, AMPAR expression at distal dendrites was not altered by either dominant negative construct (Fig. 5c,f) (tCaMKII did not affect distal GluR1 expression either; Fig. 5c, compare blue and black columns). In contrast, incubation of the slices with a low concentration of the microtubule destabilizer vincristine did significantly impair the dendritic distribution of GFP-tagged GluR2 (Fig. 5d-f). (To note, overexpression of the C-tail of Myosin Vb has been previously reported to impair GluR1 export into dendrites ${ }^{15}$; however, the tail domain used in that study contains additional sequences not present in the Myosin Vbdn globular tail used here). In conclusion, these results indicate that Myosin Va is not required for the long-range dendritic transport of AMPARs.

\section{Myosin Va transports GluR1 from dendritic shaft into spine}

To investigate the role of Myosin Va in the local transport of AMPARs into spines, we carried out confocal imaging experiments to monitor receptor distribution between spines and the adjacent dendritic shaft. Similar to the experiments described above, we 
co-expressed RFP-tagged Myosin Va or Vb dominant negatives (or plain RFP, as control) with GFP-tagged GluR1 or GluR2 subunits. The translocation of GluR1 into spines is an activity-dependent process $^{3}$, which can be mimicked by CaMKII activation $^{38}$. Therefore, in some of these experiments we expressed GFP-GluR1 together with constitutively active $\alpha \mathrm{CaMKII}$ (tCaMKII).

The accumulation of AMPARs into spines was quantified from the GFP fluorescence intensity across the spine head and the adjacent dendritic shaft ${ }^{36}$. Data are then plotted normalized to the average dendrite fluorescence (Fig. $\mathbf{6 b}, \mathbf{e}$ ) or as cumulative distributions of spine/dendrite ratios (Fig. 6c,f). As previously observed ${ }^{38}$, tCamKII significantly increased the levels of GFP-GluR1 in spines (Fig. 6b, compare ligh-gray and black columns; Fig. 6c, compare dashed and black lines). Interestingly, addition of Myosin Va-dn completely blocked this effect, to the point that GFP-GluR1 levels at spines were similar to those in the absence of tCaMKII (Fig. 6b, compare lighgray and red columns; Fig. 6c, compare dashed and red lines). Importantly, Myosin Vbdn co-expressed with GFP-GluR1 and tCamKII did not affect CamKII-mediated delivery of GluR1 receptors into spines (Fig. 6b, compare black and dark-gray columns; Fig. 6c, compare black and gray lines). Therefore, these data indicate that Myosin $\mathrm{Va}$ is required for the CaMKII-driven transport of GluR1 receptors into spines. To note, Myosin Va-dn also decreased the fraction of GFP-GluR1 present in spines in the absence of tCaMKII (Fig. 6b, compare first and last spine columns). This result would be consistent with a basal level of GluR1 delivery into spines due to spontaneous activity in the slice culture. 
To determine whether Myosin $\mathrm{Va}$ is specifically required for the regulated transport of AMPARs, we carried out similar experiments with GFP-GluR2, which is inserted into spines in a constitutive manner, independent from synaptic activity ${ }^{7,38}$. As shown in Fig. 6d-f, co-expression of Myosin Va-dn or Myosin Vb-dn did not impair the translocation of GFP-GluR2 into spines in CA1 hippocampal neurons (to note, Myosin Va-dn did produce a slight accumulation of GFP-GluR2 in spines, which was statistically significant).

These data strongly suggest that Myosin Va specifically mediates the regulated translocation of GluR1 AMPARs into spines, but it is not required for the constitutive entry of GluR2 receptors. This morphological analysis correlates very well with our electrophysiology data (Figs. 3 and 4), supporting the interpretation that Myosin $\mathrm{Va}$ is specifically engaged for the activity-dependent delivery of AMPARs into synapses, but not for their constitutive cycling.

\section{Myosin Va is not required for spine morphology}

PSD95 is an important synaptic scaffolding protein, which can associate with Myosin Va via GKAP and dynein light chain (DLC) interactions ${ }^{18}$. Our data shows that Myosin Va-dn blocks the synaptic delivery of GluR1 receptors mediated by PSD95 (Supplementary Fig. 4). Therefore, we decided to test whether Myosin Va plays a role in the targeting of PSD95 to spines. To this end, we co-expressed PSD95-GFP and RFP-Myosin Va-dn (or RFP, as control) in CA1 neurons from organotypic hippocampal slices. Distribution of PSD95-GFP in dendrites and spines was quantified as described above. As expected, PSD95 was highly concentrated in spines (Fig. 7a,b). Importantly, 
Myosin Va-dn did not alter the accumulation of PSD95-GFP into spines (Fig. 7a-c). Therefore, the blockade of PSD95-driven delivery of GluR1 to synapses by Myosin Vadn (Supplementary Fig. 4) is not due to an impairment of PSD95 targeting into spines.

It has been recently shown that other members of the myosin family, such as Myosin VI and Myosin IIB, play critical roles in spine morphology and dynamics, and consequently, in synaptic function ${ }^{14,39}$. Therefore, we tested whether Myosin Va-dn may alter spine size or density in CA1 hippocampal neurons. To this end, we co-expressed cytosolic GFP (as a volume-filling indicator) with RFP-Myosin Va-dn or with RFP as a control. GFP fluorescence across dendrites and spine heads was calculated as described above. As shown in Fig. 7d,e, GFP fluorescence in spines was not altered by Myosin Va-dn expression, indicating that spine volume is not affected by impairing Myosin Va function.

The effects of Myosin Va-dn on spine length or density were evaluated by coexpressing PSD95-GFP, as a spine marker, with RFP-Myosin Va-dn or RFP in CA1 hippocampal neurons, as described above. Confocal images were then analyzed using Neurolucida software. Spine length was determined from the peak of PSD95-GFP fluorescence to the center of the dendritic shaft, visualized from the RFP fluorescence. As shown in Fig. 7f,g, Myosin Va-dn did not alter spine length or density. Therefore, these data indicate that, in contrast to Myosin IIB and Myosin VI, Myosin Va does not play a direct role in the maintenance of spine morphology in CA1 hippocampal neurons. 


\section{Rab11 couples AMPARs with Myosin Va}

Recent studies have found that recycling endosomes are mobilized into spines ${ }^{2}$ and supply AMPARs for synaptic delivery ${ }^{40}$ during LTP. In addition, we have observed that Rab11, the small GTPase that controls recycling endosomal trafficking ${ }^{30}$, associates with AMPARs and Myosin Va (Fig. 1b,c), and is required for receptor translocation into spines during LTP $^{41}$. Therefore, Rab11-driven recycling endosomes appear to be the vesicular carriers for AMPAR delivery into spines. To determine whether Myosin $\mathrm{Va}$ is involved in the transport of these recycling endosomes, we evaluated the effect of Myosin Va-dn on the spine localization of Rab11. CA1 hippocampal neurons were co-transfected with GFP-Rab11 and RFP-Myosin Va-dn, or RFP as control. As shown in Fig. 8a,b, Myosin Va-dn produced a small, but significant, impairment in the spine accumulation of GFP-Rab11. These results suggest that Myosin $\mathrm{Va}$ is involved in the transport of recycling endosomes into spines. In addition, the modest magnitude of the shift in Rab11 spine distribution produced by Myosin Va-dn may imply that only a fraction of Rab11-containing endosomes are transported by Myosin Va.

Rab GTPases are crucial organizers of intracellular membrane trafficking in eukaryotic cells. Due to their ability to interact with multiple effectors, Rab GTPases have been proposed to act as "organelle receptors" that would couple motor proteins with specific cargo for directional membrane transport ${ }^{13,42}$. Importantly, Rab GTPases interact with motor proteins only in their active (GTP-bound) conformation ${ }^{43}$. Based on our observations on the effect of Myosin Va-dn on the transport of AMPARs and Rab11 
into spines, we tested whether Rab11 could modulate the interaction between AMPARs and Myosin Va.

To this end, we separately expressed GFP-fusion proteins of GluR1, Myosin Va globular tail (containing the cargo binding domain) and Rab11 in BHK cells. Whole-cell extracts in the presence of detergent were then prepared, and mixed in different combinations to test the effect of Rab proteins on the interaction between GluR1 and Myosin Va. The association between GluR1 and Myosin Va was evaluated by coimmunoprecipitation using an anti-GluR1 antibody, in the presence of GDP or a nonhydrolysable GTP-analog (GMP-PNP; Sigma). The specificity of the interaction was evaluated by substituting GFP-GluR1 with GFP. As shown in Fig. 8c (representative example of four independent experiments), the association between Myosin Va and GluR1 was weak but detectable in the absence of added Rab protein (compare lanes 1 and 2). Interestingly, this interaction was enhanced by adding Rab11 (compare lanes 2 and 4), but only in the presence of non-hydrolysable GTP (compare lanes 4 with 6). These results indicate that active Rab11 (GTP-bound) facilitates Myosin Va binding to GluR1, suggesting that Rab11 may act as the vesicular link that couples AMPARs and Myosin Va during their transport into spines. 


\section{DISCUSSION}

In this study we show that Myosin $\mathrm{Va}$, an actin-based motor protein present at synaptic sites, mediates the transport of organelles that carry AMPARs into spines in an activity-dependent manner. Using an unbiased biochemical approach, we identified Myosin Va as an interacting partner for AMPARs in hippocampal neurons. We also determined that this association occurs through a direct interaction between the GluR1 C-terminus and the globular tail of Myosin Va, which is the cargo binding domain of the motor protein. Using electrophysiological assays on hippocampal slices, we determined that Myosin Va function is required for LTP and for GluR1 synaptic delivery triggered by CaMKII activation or by PSD95 overexpression. Importantly, the synaptic trafficking of AMPARs appears to be specifically carried out by Myosin Va, since blockade of other myosin isoforms, such as Myosin $\mathrm{Vb}$ and Myosin $\mathrm{VI}$, did not alter AMPAR-mediated synaptic transmission. In addition, using confocal fluorescence imaging, we find that Myosin Va mediates a very precise transport step: the short-range translocation of GluR1-containing AMPARs from the dendritic shaft into the spine head. In contrast, Myosin $\mathrm{Va}$ is nor required for the long-range trafficking of receptors along dendrites, nor

for the constitutive entry of GluR2 receptors into spines. And finally, we have identified the small GTPase Rab11 as a vesicular carrier that couples Myosin Va with its AMPAR cargo. Therefore, these combined results support the notion that Myosin Va is a critical player in the regulation of synaptic activity, which may be related to the cognitive and neurological deficits associated to Myosin Va mutations in humans. 
It is worth noting that a previous study did not find any deficits in hippocampal synaptic function or plasticity in a spontaneous mouse mutant lacking Myosin Va (dilutelethal) ${ }^{27}$. However, our data using both dominant negative approaches and siRNA knock-down indicates that Myosin Va is required for LTP in CA1 hippocampal neurons. We cannot be certain of the reason for this discrepancy, but it may be related with the acute blockade of Myosin Va function in our system (15 hours for dominant negative expression or 3 days for RNA interference) versus the chronic absence of Myosin Va during development and postnatal life in the dilute-lethal mice.

A critical conclusion from our work is that Myosin $\mathrm{Va}$ is specifically required for the regulated transport of AMPA receptors, but not for their constitutive (activityindependent) trafficking. Myosin Va activity is tightly controlled by intracellular calcium levels ${ }^{44}$. In the absence of calcium, Myosin Va adopts a compact, inactive conformation in which the cargo binding domain folds back on the motor domain, rendering Myosin Va incompetent for transport. Upon calcium binding, Myosin Va undergoes a large conformational change, opening into an extended, active configuration ${ }^{44}$. This calcium regulation of Myosin $\mathrm{Va}$ activity is obviously reminiscent of the long-established requirement of postsynaptic calcium for LTP expression ${ }^{45}$. Therefore, it is tempting to speculate that the rise in intracellular calcium upon NMDAR activation may constitute a direct link between LTP induction and the triggering of Myosin Va-dependent organelle transport. Alternatively, the transport machinery may stay constitutively active while LTP regulates the competency of GluR1 to interact with it (perhaps through GluR1 phosphorylation and/or binding to adaptor proteins) (see model in Supplementary Fig. 5). 
Here we propose that Rab11 mediates the association of Myosin Va with its AMPAR cargo. The concept of Rab proteins as organelle acceptors that couple specific motor proteins to cargo vesicles is not new ${ }^{13,42}$. However, this is the first time that the Rab11-Myosin Va pairing is reported. In fact, the motor protein typically associated to Rab11-dependent trafficking is Myosin $\mathrm{Vb}^{32}$, as it has been described for the export of AMPARs from the cell soma into dendrites ${ }^{15}$. However, our combined electrophysiological and imaging data indicate that Myosin $\mathrm{Vb}$ is not involved in the synaptic transport of GluR1. The unexpected coupling between Myosin Va and Rab11 that we describe may be a functional consequence of the recruitment of Rab11-driven endosomes in activity-dependent trafficking within spines ${ }^{2,41}$, together with the specialization of Myosin Va for acute, regulated exocytic transport.

Finally, several mechanochemical properties of Myosin Va make it an ideal motor to mediate the specific transport event we are proposing here. First, receptor insertion into spines implies a switch from the microtubular cytoskeleton present along dendrites towards the actin filaments enriched in spines. Interestingly, Myosin Va is able to bridge across microtubule and actin cytoskeletons by virtue of separate interactions with actin (through its $\mathrm{N}$-terminal motor domain) and with tubulin (through its $\mathrm{C}$-terminal cargo binding domain $)^{46}$. In fact, Myosin $\mathrm{Va}$ has been shown to passively diffuse along microtubules ${ }^{47}$. In agreement with this transitional role between different cytoskeletal elements, the cargo binding domain of Myosin Va directly binds kinesin ${ }^{31,48}$, a microtubule-dependent motor protein. Second, Myosin Va is flexible enough to step over or to switch filaments at intersections and branching points in the actin cytoskeleton ${ }^{47}$. This property should facilitate maneuvering cargo across the intricate 
cytoskeletal meshwork of the spine. And third, Myosin Va is a processive motor, in contrast for example, to Myosin $\mathrm{II}^{49}$. The dimensions of a single processive run by Myosin Va (40 to 50 steps, approximately $1.5 \mu \mathrm{m}$ ) fit very well with the average spine length, and therefore with the travel distance required to transport organelles from the dendritic shaft into the spine head.

In conclusion, this work offers new insights into the molecular machinery that organizes membrane trafficking at postsynaptic terminals, and proposes a specific motor protein (Myosin $\mathrm{Va}$ ) and its organelle acceptor (Rab11) to drive the directional transport of AMPARs during synaptic plasticity. 


\section{METHODS}

Constructs of recombinant proteins and expression

The C-terminal sequences (globular tails) of mouse Myosin Va (amino acids 1473 to 1880), rat Myosin Vb (amino acids 1436 to 1846) and human Myosin VI (amino acids 835 to 1285) were cloned upstream of the coding sequence for EGFP (Clontech) or a red fluorescent protein variant $\left(\right.$ tdimer $2^{36}$ ). Myosin Va GST constructs were generated in pGEX4T-3 using amino acids 1396-1830 (globular tail) and 1152-1395 (media tail) from rat Myosin Va. The GFP-tagged AMPAR subunits, PSD95 and the truncated CaMKII constructs were made as previously described ${ }^{7,37}$. The anti-Myosin Va small interference RNA (MyoVa siRNA) corresponds to nucleotides 5344 to 5362 in the rat Myosin Va mRNA (accession number AB035736). A scrambled siRNA was used as control (GGTTTCGTACTTTCTCTTA). siRNAs were cloned into pSuper vector for expression in COS-7 cells and neurons. Organotypic cultures of hippocampal slices were prepared from P5-6 rats and recombinant proteins were expressed using Sindbis virus or biolistic gene delivery ("gene gun"; Bio-Rad) ${ }^{36}$. Protein expression was typically for 14 hours or

for 2.5 days when expressing AMPAR subunits. siRNAs were expressed in hippocampal slices for 3-4 days. All biosafety procedures and animal care protocols were approved by the University of Michigan Committee on Use and Care of Animals (UCUCA).

\section{Co-immunopurification and GST pull-downs}

Hippocampal neurons plated on coverslips coated with poly-D-lysine $(30 \mu \mathrm{g} / \mathrm{ml})$ and laminin $(2 \mu \mathrm{g} / \mathrm{ml})$ at a density of 300,000/well were washed once with PBS (containing 1 $\mathrm{mM} \mathrm{MgCl}$ and $0.1 \mathrm{mM} \mathrm{CaCl}_{2}$ ) and incubated for 1 hour at $4^{\circ} \mathrm{C}$ with a buffer containing 
$50 \mathrm{mM}$ Tris- $\mathrm{HCl}, 150 \mathrm{mM} \mathrm{NaCl}, 1 \mathrm{mM}$ EDTA, 1\% Triton X-100, 1\% saponin and a protease inhibitor cocktail ("Complete EDTA-free", Roche). For immunopurification experiments the soluble extracts of neurons were loaded on a column of $\mathrm{CNBr}$ Sepharose 4B resin bound to polyclonal antibody against GluR2 subunit and incubated overnight at $4^{\circ} \mathrm{C}$. The column was washed with PBS (0.1\% saponin, $0.1 \%$ Triton $\left.\mathrm{X}-100\right)$. The complexes associated to GluR2 were eluted with $0.2 \mathrm{M}$ glycin, $\mathrm{pH}$ 2.2. The coimmunoprecipitation experiments from neurons were performed as described ${ }^{50}$. For GST pulldown experiments, $200 \mu \mathrm{g}$ of soluble extracts were incubated with glutathione sepharose 4B (Amersham Pharmacia Biotech) coupled to 5-10 $\mu \mathrm{g}$ of GST or GST fusion (approximately $5 \mu \mathrm{l}$ of bed volume) for $2 \mathrm{hr}$ at $4^{\circ} \mathrm{C}$, followed by four washes in PBS (0.1\% Triton $\mathrm{X}-100)$. These samples were then washed and immunoprecipitated proteins were eluted by boiling in $1 x$ Laemmli sample buffer and separated by SDSPAGE. Visualization of immunoprecipitated proteins was done by Western Blot with antiGluR1, -GluR2/3,-Myosin Va, -myc, -GFP or -His antibodies.

\section{Electrophysiology}

Voltage-clamp whole cell recordings were obtained from nearby infected and uninfected CA1 pyramidal neurons, under visual guidance using fluorescence and transmitted light illumination. External solution contained $119 \mathrm{mM} \mathrm{NaCl}, 2.5 \mathrm{mM} \mathrm{KCl}, 1$ $\mathrm{mM} \mathrm{NaH} \mathrm{PO}_{4}, 11 \mathrm{mM}$ glucose, $26 \mathrm{mM} \mathrm{NaHCO}_{3}, 4 \mathrm{M} \mathrm{MgCl}_{2}, 4 \mathrm{mM} \mathrm{CaCl} 2$ and $2 \mu \mathrm{M} 2-$ chloroadenosine, at $\mathrm{pH} 7.4$, and was gassed with $95 \% \mathrm{O}_{2}$ and $5 \% \mathrm{CO}_{2}$. Patch recording pipettes (4-7 M $\Omega$ ) were filled with internal solution containing $115 \mathrm{mM} \mathrm{CsMeSO}{ }_{3}, 20 \mathrm{mM}$ CsCl, 10 mM HEPES, 2.5 mM MgCl 2,4 mM Na2-ATP, 0.4 mM Na-GTP, 10 mM sodium 
phosphocreatine, $0.6 \mathrm{mM}$ EGTA and $0.1 \mathrm{mM}$ spermine, $\mathrm{pH}$ 7.25. In experiments with intracellular perfusion of peptides (pep2m or pep4c, Tocris; $1 \mathrm{mM}$ final concentration), the internal solution was supplemented with protease inhibitors leupeptin and pepstatin (100 $\mu \mathrm{M}$ each). Bipolar stimulating electrodes were placed over Schaffer collateral fibers between 250 and $300 \mu \mathrm{m}$ from the CA1 recorded cell, and synaptic responses were evoked with single voltage pulses (200 $\mu$ s, up to $30 \mathrm{~V})$. Synaptic AMPAR-mediated responses were acquired at $-60 \mathrm{mV}$. NMDAR responses were recorded at $+40 \mathrm{mV}$ at a latency at which AMPAR responses have fully decayed (60 ms after stimulation). In both cases, $100 \mu \mathrm{M}$ picrotoxin was present in the external solution. $\mathrm{GABA}_{\mathrm{A}}$ responses were acquired at $0 \mathrm{mV}$ in the absence of receptor antagonists; therefore, they should be considered as a combination of mono- and disynaptic IPSCs. For rectification studies, AMPAR responses were recorded at $-60 \mathrm{mV}$ and $+40 \mathrm{mV}$ in the presence of $0.1 \mathrm{mM}$ DL-2-amino-5-phosphonovaleric acid in the external solution. LTP was induced using a pairing protocol, by stimulating Schaffer collateral fibers at $3 \mathrm{~Hz}$ for $1.5 \mathrm{~min}$, while depolarizing the postsynaptic cell at $0 \mathrm{mV}$. Because only CA1 cells (and not CA3 cells) are infected, this configuration ensures that recombinant proteins are always expressed exclusively in the postsynaptic cell. Whole-cell recordings were made with a Multiclamp 700A amplifier (Axon Instruments).

\section{Fluorescence imaging}

Immunofluorescence and imaging experiments were carried out as described in the Supplementary Information and ${ }^{36}$. 
In vitro binding of AMPARs, Myosin Va and Rab11

BHK cells were infected with GFP tagged Myosin Va-dn, Rab11, GluR1 or soluble GFP. Cells were then homogeneized in $50 \mathrm{mM}$ Tris- $\mathrm{HCl}, \mathrm{pH}$ 7.4, $150 \mathrm{mM} \mathrm{NaCl}, 10 \mathrm{mM}$ ATP, $10 \mathrm{mM} \mathrm{NaF}, 1 \mu \mathrm{M}$ Microcystin LR, $0.5 \mu \mathrm{M}$ Calyculin A, $10 \mathrm{mM}$ EDTA, $0.1 \mathrm{mM}$ PMSF, $2 \mu \mathrm{g} / \mathrm{ml}$ CLAP (cocktail of Chymostatin, Leupeptin, Pepstatin A and Antipain), 0.5\% Sodium Deoxycholate and 1\% Nonidet P-40. Extracts were then mixed in the presence of $30 \mathrm{mM} \mathrm{MgCl}$, and $0.1 \mathrm{mM} \mathrm{GMP-PNP}$ or $1 \mathrm{mM} \mathrm{GDP}$, as indicated. Immunoprecipitates were obtained by incubation of $2 \mu \mathrm{g}$ of anti-GluR1 polyclonal antibody with $60 \mu \mathrm{l}$ of protein G-sepharose beads (50\%), for 4 hours at $4^{\circ} \mathrm{C}$. These samples were then washed and immunoprecipitated proteins were eluted by boiling in 1x Laemmli sample buffer and separated by SDS-PAGE. Visualization of immunoprecipitated proteins was done by Western Blot with anti-GFP antibodies (Roche).

Statistical analyses

Statistical significance was determined by the Kolmogorof-Smirnov test for cumulative distributions. When comparing mean values, statistical significance was determined by the Mann-Whitney test if only two distributions are being compared, or by ANOVA followed by a Kruskal-Wallis test, if multiple distributions are analyzed. 


\section{AUTHOR CONTRIBUTION}

S.S.C. is responsible for most of the experimental work and wrote part of the manuscript. S.B carried out the biochemical experiments of Fig. 1 and the immunocytochemistry in Fig. 2a,b. T.C.B. contributed some of the electrophysiology and imaging experiments. M.-F.L. developed and characterized the siRNA used in this study. D.S.B. carried out some of the initial cloning of this project. A.E.-H., M.P. and J.A.E. designed and supervised the experiments carried out by S.B., M.-F.L. and S.S.C., respectively. J.A.E. coordinated the study and wrote most of the manuscript.

\section{ACKNOWLEDGEMENTS}

We thank Vladimir Gelfand and Ron Holz for the plasmid containing the mouse Myosin Va globular tail; David Wells and Mark Mooseker for the human Myosin VI; Yoshiko Takagishi for the chicken full-length Myosin Va; Sang Lee for letting us test an unpublished siRNA against Myosin Va (different from the one shown in this study); and Robert Malinow for the PSD95 construct. We also thank Lois Weisman, Miriam Meisler and Ron Holz for their comments on this work. This work was supported by grants from the National Institute of Mental Health (MH070417 to J.A.E. and F31-MH070205 to T.C.B.), Fundação para a Ciência e a Tecnologia (SFRH/BPD/14620 to S.S.C.) and Telethon-Italy (SYNSCAF project to M.P.). 


\section{REFERENCES}

1. Kennedy, M.J. \& Ehlers, M.D. Organelles and trafficking machinery for postsynaptic plasticity. Annu Rev Neurosci 29, 325-62 (2006).

2. Park, M. et al. Plasticity-induced growth of dendritic spines by exocytic trafficking from recycling endosomes. Neuron 52, 817-30 (2006).

3. Shi, S.H. et al. Rapid spine delivery and redistribution of AMPA receptors after synaptic NMDA receptor activation. Science 284, 1811-6 (1999).

4. Kim, J., Jung, S.C., Clemens, A.M., Petralia, R.S. \& Hoffman, D.A. Regulation of dendritic excitability by activity-dependent trafficking of the a-type $k(+)$ channel subunit kv4.2 in hippocampal neurons. Neuron 54, 933-47 (2007).

5. Shepherd, J.D. \& Huganir, R.L. The cell biology of synaptic plasticity: AMPA receptor trafficking. Annu Rev Cell Dev Biol 23, 613-43 (2007).

6. Passafaro, M., Piech, V. \& Sheng, M. Subunit-specific temporal and spatial patterns of AMPA receptor exocytosis in hippocampal neurons. Nat Neurosci 4, 917-26 (2001).

7. Shi, S., Hayashi, Y., Esteban, J.A. \& Malinow, R. Subunit-specific rules governing AMPA receptor trafficking to synapses in hippocampal pyramidal neurons. Cell 105, 331-43 (2001).

8. Luscher, C. et al. Role of AMPA receptor cycling in synaptic transmission and plasticity. Neuron 24, 649-58 (1999).

9. Nishimune, A. et al. NSF binding to GluR2 regulates synaptic transmission. Neuron 21, 87-97 (1998). 
10.Song, I. et al. Interaction of the N-ethylmaleimide-sensitive factor with AMPA receptors. Neuron 21, 393-400 (1998).

11. Hayashi, Y. et al. Driving AMPA receptors into synapses by LTP and CaMKII: requirement for GluR1 and PDZ domain interaction. Science 287, 2262-7 (2000).

12. Carlisle, H.J. \& Kennedy, M.B. Spine architecture and synaptic plasticity. Trends Neurosci 28, 182-7 (2005).

13. Karcher, R.L., Deacon, S.W. \& Gelfand, V.I. Motor-cargo interactions: the key to transport specificity. Trends Cell Biol 12, 21-7 (2002).

14. Osterweil, E., Wells, D.G. \& Mooseker, M.S. A role for myosin VI in postsynaptic structure and glutamate receptor endocytosis. J Cell Biol 168, 329-38 (2005).

15. Lise, M.F. et al. Involvement of myosin $\mathrm{Vb}$ in glutamate receptor trafficking. J Biol Chem 281, 3669-78 (2006).

16. Triller, A. \& Choquet, D. Surface trafficking of receptors between synaptic and extrasynaptic membranes: and yet they do move! Trends Neurosci 28, 133-9 (2005).

17. Mercer, J.A., Seperack, P.K., Strobel, M.C., Copeland, N.G. \& Jenkins, N.A. Novel myosin heavy chain encoded by murine dilute coat colour locus. Nature $\mathbf{3 4 9}, 709-13$ (1991).

18. Naisbitt, S. et al. Interaction of the postsynaptic density-95/guanylate kinase domainassociated protein complex with a light chain of myosin- $\mathrm{V}$ and dynein. $\mathrm{J}$ Neurosci $\mathbf{2 0}$, 4524-34 (2000).

19. Walikonis, R.S. et al. Identification of proteins in the postsynaptic density fraction by mass spectrometry. J Neurosci 20, 4069-80 (2000). 
20. Yoshimura, A. et al. Myosin-Va facilitates the accumulation of mRNA/protein complex in dendritic spines. Curr Biol 16, 2345-51 (2006).

21. Pastural, E. et al. Griscelli disease maps to chromosome $15 q 21$ and is associated with mutations in the myosin-Va gene. Nat Genet 16, 289-92 (1997).

22. Sanal, O. et al. An allelic variant of Griscelli disease: presentation with severe hypotonia, mental-motor retardation, and hypopigmentation consistent with Elejalde syndrome (neuroectodermal melanolysosomal disorder). J Neurol 247, 570-2 (2000).

23.Tabb, J.S., Molyneaux, B.J., Cohen, D.L., Kuznetsov, S.A. \& Langford, G.M. Transport of ER vesicles on actin filaments in neurons by myosin V. J Cell Sci $\mathbf{1 1 1}$ ( Pt 21), 3221-34 (1998).

24. Takagishi, Y. et al. The dilute-lethal (dl) gene attacks a Ca2+ store in the dendritic spine of Purkinje cells in mice. Neurosci Lett 215, 169-72 (1996).

25. Petralia, R.S. et al. Glutamate receptor targeting in the postsynaptic spine involves mechanisms that are independent of myosin Va. Eur J Neurosci 13, 1722-32 (2001).

26. Miyata, M. et al. Local calcium release in dendritic spines required for long-term synaptic depression. Neuron 28, 233-44 (2000).

27. Schnell, E. \& Nicoll, R.A. Hippocampal synaptic transmission and plasticity are preserved in myosin Va mutant mice. J Neurophysiol 85, 1498-501 (2001).

28. Van Zundert, B., Yoshii, A. \& Constantine-Paton, M. Abnormally large AMPA receptor currents in flailer mutant mice. Society for Neuroscience Abstract 493.20(2005). 
29. Wenthold, R.J., Petralia, R.S., Blahos, J., II \& Niedzielski, A.S. Evidence for multiple AMPA receptor complexes in hippocampal CA1/CA2 neurons. J Neurosci 16, 19829 (1996).

30. Ullrich, O., Reinsch, S., Urbe, S., Zerial, M. \& Parton, R.G. Rab11 regulates recycling through the pericentriolar recycling endosome. J Cell Biol 135, 913-24 (1996).

31. Brown, J.R., Simonetta, K.R., Sandberg, L.A., Stafford, P. \& Langford, G.M. Recombinant globular tail fragment of myosin- $V$ blocks vesicle transport in squid nerve cell extracts. Biol Bull 201, 240-1 (2001).

32. Lapierre, L.A. et al. Myosin vb is associated with plasma membrane recycling systems. Mol Biol Cell 12, 1843-57 (2001).

33. Rodriguez, O.C. \& Cheney, R.E. Human myosin-Vc is a novel class $V$ myosin expressed in epithelial cells. J Cell Sci 115, 991-1004 (2002).

34.Zhu, J.J., Esteban, J.A., Hayashi, Y. \& Malinow, R. Postnatal synaptic potentiation: delivery of GluR4-containing AMPA receptors by spontaneous activity. Nat Neurosci 3, 1098-106 (2000).

35. Watt, A.J., van Rossum, M.C., MacLeod, K.M., Nelson, S.B. \& Turrigiano, G.G. Activity coregulates quantal AMPA and NMDA currents at neocortical synapses. Neuron 26, 659-70 (2000).

36. Gerges, N.Z., Brown, T.C., Correia, S.S. \& Esteban, J.A. Analysis of Rab protein function in neurotransmitter receptor trafficking at hippocampal synapses. Methods Enzymol 403, 153-66 (2005). 
37.Ehrlich, I. \& Malinow, R. Postsynaptic density 95 controls AMPA receptor incorporation during long-term potentiation and experience-driven synaptic plasticity. J Neurosci 24, 916-27 (2004).

38. Piccini, A. \& Malinow, R. Critical postsynaptic density 95/disc large/zonula occludens-1 interactions by glutamate receptor 1 (GluR1) and GluR2 required at different subcellular sites. J Neurosci 22, 5387-92 (2002).

39. Ryu, J. et al. A critical role for myosin Ilb in dendritic spine morphology and synaptic function. Neuron 49, 175-82 (2006).

40.Park, M., Penick, E.C., Edwards, J.G., Kauer, J.A. \& Ehlers, M.D. Recycling endosomes supply AMPA receptors for LTP. Science 305, 1972-5 (2004).

41.Brown, T.C., Correia, S.S., Petrok, C.N. \& Esteban, J.A. Functional compartmentalization of endosomal trafficking for the synaptic delivery of AMPA receptors during long-term potentiation. J Neurosci 27, 13311-5 (2007).

42. Seabra, M.C. \& Coudrier, E. Rab GTPases and myosin motors in organelle motility. Traffic 5, 393-9 (2004).

43. Jordens, I., Marsman, M., Kuijl, C. \& Neefjes, J. Rab proteins, connecting transport and vesicle fusion. Traffic 6, 1070-7 (2005).

44. Krementsov, D.N., Krementsova, E.B. \& Trybus, K.M. Myosin V: regulation by calcium, calmodulin, and the tail domain. J Cell Biol 164, 877-86 (2004).

45. Lynch, G., Larson, J., Kelso, S., Barrionuevo, G. \& Schottler, F. Intracellular injections of EGTA block induction of hippocampal long-term potentiation. Nature 305, 719-21 (1983). 
46. Cao, T.T., Chang, W., Masters, S.E. \& Mooseker, M.S. Myosin-Va binds to and mechanochemically couples microtubules to actin filaments. Mol Biol Cell 15, 151-61 (2004).

47. Ali, M.Y. et al. Myosin Va maneuvers through actin intersections and diffuses along microtubules. Proc Natl Acad Sci U S A 104, 4332-6 (2007).

48. Huang, J.D. et al. Direct interaction of microtubule- and actin-based transport motors. Nature 397, 267-70 (1999).

49. Mehta, A.D. et al. Myosin- $\mathrm{V}$ is a processive actin-based motor. Nature 400, 590-3 (1999).

50. Dunah, A.W. et al. LAR receptor protein tyrosine phosphatases in the development and maintenance of excitatory synapses. Nat Neurosci 8, 458-67 (2005). 


\section{FIGURE LEGENDS}

Figure 1. Myosin Va associates with AMPA receptors through the GluR1 Cterminus. a. Silver staining of AMPARs and co-immunopurified proteins from hippocampal neurons. Immunopurification was carried out with an anti-GluR2 Cterminus antibody or with a non-immune IgG, as control. Myosin Va was identified by mass spectroscopy. b. Coimmunoprecipitation between AMPARs and associated proteins using anti-GluR1 and -GluR2 antibodies. Non-immune IgG and anti-NR1 antibodies were used as control. c. Left: Western blot analysis of AMPA receptor subunits from hippocampal neurons pulled-down with the medial ("GST-M") or globular ("GST-G") tail of Myosin Va fused to GST. Plain GST was used as control. Middle: Similar GST pull-downs using extracts from COS-7 cells expressing full-length myctagged GluR1, a myc-tagged GluR1 mutant lacking the last 30 amino acids, or EGFPtagged Rab11, as indicated. Anti-myc antibodies were used to detect recombinant GluR1, and anti-GFP for recombinant Rab11. Right: His-tagged GluR1 C-terminus (last 50 amino acids) was expressed in COS-7 cells and purified through a nickel-column. The purified recombinant protein was used for pull-down with GST or GST-Myosin Va globular tail. Anti-His antibodies were used to detect pulled-down GluR1 C-terminus. Lower panels: Coomassie staining of GST fusion proteins used in the pull-downs.

Figure 2. Subcellular distribution of Myosin $\mathrm{Va}$ in neurons and partial colocalization with synaptic proteins. a, b. Co-immunofluorescence labeling of GluR2 (A) and GluR1 (B) (left panels) with Myosin Va (middle panels) in primary 
hippocampal neurons. Overlay in right panels. Higher magnification of dendritic branches in lower panels. c, d. Co-immunofluorescence labeling of PSD95 (C) and actin (D) (left panels) with Myosin Va (middle panels) in hippocampal slices. Overlay in right panels. Smaller panels on the right show higher magnification images of dendritic branches. Scale bars represent $2 \mu \mathrm{m}$.

Figure 3. Myosin Va-dn-mediated depression of AMPA and NMDA currents is dependent on spontaneous activity. Organotypic slice cultures were infected with GFP-tagged Myosin Va-dn or Myosin VI-dn, as indicated. Some slices were incubated in $12 \mathrm{mM} \mathrm{MgCl}$ ("high $\mathrm{Mg}^{2+»)}$ or $1 \mu \mathrm{M}$ tetrodotoxin ("TTX") during the expression of the recombinant protein to block spontaneous activity, as indicated. Double whole-cell recordings were established from pairs of uninfected and infected CA1 neurons under voltage-clamp configuration. The amplitude of the evoked postsynaptic response mediated by AMPA (a), NMDA (b) or $\mathrm{GABA}_{\mathrm{A}}$ receptors (c) was normalized to the uninfected neurons for each experimental condition and plotted as averages \pm standard error of the mean. d. Presynaptic function was evaluated by monitoring paired-pulse facilitation at different interstimulus intervals $(50,100,200$, and $400 \mathrm{~ms})$ in uninfected neurons and neurons expressing GFP-Myosin Va-dn. Paired-pulse facilitation was calculated as the ratio between the amplitude of the second response versus the amplitude of the first response. Insets, Sample trace of evoked AMPA receptormediated synaptic responses with an interstimulus interval of $100 \mathrm{~ms}$. Error bars represent standard error of the mean in all figures. " $n$ " represents the number of cells. 
Statistical significance was determined by using the Wilcoxon test for paired data (a-c) and by the Mann-Whitney test for unpaired data (d).

Figure 4. Myosin Va does not participate in constitutive receptor cycling, but it is required for LTP and CaMKII-mediated synaptic delivery of GluR1. a. Time course of AMPAR-mediated responses recorded from CA1 neurons expressing Myosin Va-dn (solid symbols) or uninfected neurons (empty symbols), during whole-cell pipette infusion of pep2m. Responses are normalized to a 2 min baseline from the beginning of the recording. Inset: sample traces from baseline (thick lines) and 25-30 min average (thin lines). b. Average remaining current after $25-30$ min recording from the time courses shown in a, and from similar experiments in which the control peptide, pep4c, was used. $P$ values indicate statistical significance with respect to a 2 min baseline from the beginning of the recordings. c. LTP was induced in CA1 neurons infected with virus expressing GFP-Myosin Va-dn (black squares), transfected with anti-Myosin Va siRNA (grey squares) or untransfected neurons (empty symbols). Slices were incubated in 12 $\mathrm{mM} \mathrm{MgCl}_{2}$ or $1 \mu \mathrm{M}$ tetrodotoxin during transfection to prevent depression of basal AMPAR and NMDAR responses (similar results were obtained with both treatments, and the data were pooled together). Inset, sample trace of evoked AMPA receptormediated synaptic responses recorded at $-60 \mathrm{mV}$ before pairing (thick line) and $30 \mathrm{~min}$ after pairing (thin line). d. Normalized average potentiation of AMPAR-mediated responses collected between 25 and 30 min of the time course shown in c. Paired (LTP) and unpaired (control) pathways are shown. e. AMPAR mediated responses were recorded at $-60 \mathrm{mV}$ and $+40 \mathrm{mV}$. The rectification index $(\mathrm{RI})$ was calculated as the ratio 
of responses at these holding potentials. Sample traces are shown above the corresponding columns of the plot. f. Similar experiments as the one shown in e were carried out using a specific siRNA against Myosin Va or a scrambled (control) siRNA. Sample traces are shown above the corresponding columns of the plot.

Figure 5. Myosin $\mathrm{Va}$ is not required for the trafficking of AMPA receptors into distal apical dendrites. Hippocampal slices in culture were co-transfected with GFPGluR1 (a-c) or GFP-GluR2 (D-F), together with tCaMKII and RFP-tagged Myosin Va-dn or Myosin Vb-dn, as indicated. a, d. Representative confocal images of transfected CA1 neurons. b, e. GFP-AMPAR fluorescence intensity along the primary apical dendrite was normalized to the maximum fluorescence (peak of fluorescence observed in the soma of neuron) and plotted as a function of the distance from the cell body. Average values are represented for each condition (number of analyzed cells is indicated in c and f). c, f. Normalized average fluorescence at distal apical dendrites calculated between $100 \mu \mathrm{m}$ and $200 \mu \mathrm{m}$ from the soma, for GFP-GluR1 (c) and GFP-GluR2 (f) transfected cells. Some GFP-GluR2 cells were treated with $50 \mathrm{nM}$ vincristine during the expression time (this treatment does not affect the dendritic distribution of cytosolic RFP; not shown). " $n$ " represents number of cells. Statistical significance was calculated with Mann-Whitney test.

Figure 6. Myosin Va-dn specifically impairs the translocation of GluR1 receptors from dendrites into spines. a-c. Hippocampal CA1 neurons were transfected with GFP-GluR1 or GFP-GluR1 plus tCamKII, together with RFP, RFP-Myosin Va-dn or 
RFP-Myosin Vb-dn, as indicated. a. Representative confocal images of spines (arrows) and the adjacent dendritic shafts from neurons transfected with GFP-GluR1 and tCaMKII, together with RFP (top) or RFP-Myosin Va-dn (bottom), as indicated. Scale bar: $1 \mu \mathrm{m}$. b. Average GFP-GluR1 fluorescence intensity in spines was normalized to GFP fluorescence in the adjacent dendrite for each experimental condition. " $\mathrm{n}$ " represents number of spine-dendrite pairs from 10 neurons in each condition. c. Cumulative distributions of spine/dendrite ratios from the same data plotted in b: "GFPGluR1 + RFP" (dashed line), "GFP-GluR1 + tCaMKII + RFP" (black line), "GFP-GluR1 + tCaMKII + RFP-Myosin Va-dn" (red line), "GFP-GluR1 + tCaMKII + RFP-Myosin Vb-dn" (grey line). Statistical significance is expressed with respect to the "GFP-GluR1 + tCaMKII + RFP" distribution, according to the Kolmogorov-Smirnov test. d, e. Similar to a, b, with CA1 hippocampal neurons transfected with GFP-GluR2 together with RFP, RFP-Myosin Va-dn or RFP-Myosin Vb-dn, as indicated. "n" represents number of spinedendrite pairs from 10 (RFP) or 11 (RFP-Myosin-dn) neurons. f. Cumulative distributions of spine/dendrite ratios from the same data plotted in e: "GFP-GluR2 + RFP" (black line), "GFP-GluR2 + RFP-Myosin Va-dn" (red line), "GFP-GluR2 + RFPMyosin Vb-dn" (grey line). Statistical significance is expressed with respect to the "GFPGluR2 + RFP" distribution, according to the Kolmogorov-Smirnov test.

Figure 7. Myosin Va is not required for the trafficking of PSD95 into spines or for spine morphology. a-c. Hippocampal CA1 neurons were transfected with PSD95-GFP together with RFP or RFP-Myosin Va-dn. a. Representative confocal images of dendritic branches from neurons transfected with PSD95-GFP plus RFP (left) or RFP-Myosin Va- 
dn (right), as indicated. Scale bar: $5 \mu \mathrm{m}$. b. Average PSD95-GFP fluorescence intensity in spines was normalized to GFP fluorescence in adjacent dendrite for each experimental condition, as indicated. c. Cumulative distributions of spine/dendrite ratios from the same data plotted in $\mathbf{b}$. Statistical significance was calculated according to the Kolmogorov-Smirnov test. "n" represent number of spine-dendrite pairs. d, e. Similar to a, c, with CA1 hippocampal neurons co-transfected with GFP together with RFP or RFP-Myosin Va-dn, as indicated. GFP is used as a volume indicator. "n" represents number of spine-dendrite pairs. Scale bar: $1 \mu \mathrm{m} . \mathbf{f}, \mathbf{g}$. Spine length (f) and spine density (g) were determined using PSD95-GFP fluorescence as a spine marker. " $\mathrm{n}$ " is the number of spines (f) or dendritic branches $(\mathbf{g})$. Statistical significance was determined by the Mann-Whitney test for unpaired data.

Figure 8. Myosin Va-dn impairs the translocation of Rab11 from dendrites into spines and Rab11 facilitates the interaction of GluR1 with the Myosin Va globular tail. a. Representative confocal images of CA1 hippocampal neurons transfected with GFP-Rab11 and RFP (top) or GFP-Rab11 plus RFP-Myosin Va-dn (bottom). Scale bar:

$2 \mu \mathrm{m}$. b. GFP-Rab11 fluorescence was quantified and plotted as cumulative distributions of spine/dendrite ratios of GFP fluorescence, as described for Figs. 6 and 7. " $\mathrm{n}$ " represent number of spine-dendrite pairs from 10 neurons in each condition. Statistical significance was calculated using the Kolmogorov-Smirnov test. c. Whole extracts of BHK cells separately expressing GFP, GFP-Rab11, GFP-GluR1 and GFPMyosin Va globular tail (GFP-MyoVa-GT) were combined, as indicated (GFP-MyoVaGT was present in all combinations). Proteins were incubated in the presence of non- 
hydrolizable GTP (GMP-PNP; Sigma) or GDP, and immunoprecipitated with an antiGluR1 antibody. Samples were then analyzed by western blotting with an anti-GFP antibody. The presence of GFP, GFP-GluR1 and GFP-Myosin Va-GT in the immunoprecipitated fraction is indicated in the figure. Western blot shows a representative example from 4 independent experiments. 
a. Immunopurification

(Silver staining)

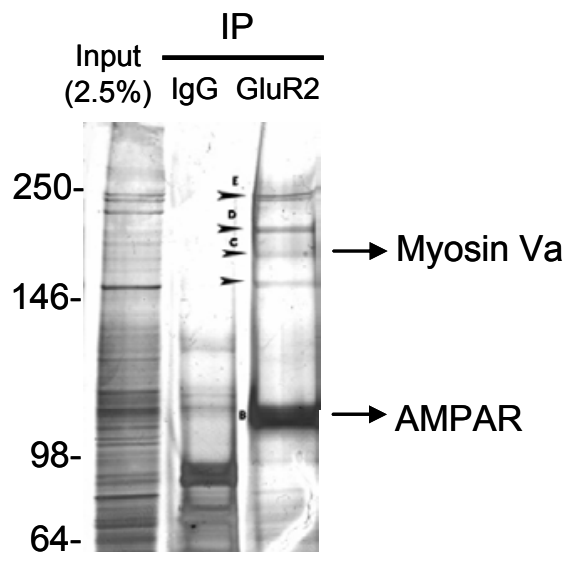

c. GST pull-down b. Immunoprecipitation

(Western blot)
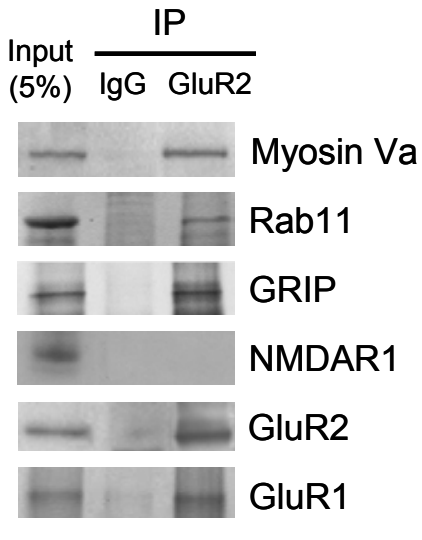

$\underset{(5 \%)}{\text { Input }} \frac{\text { IPG GluR1 }}{\lg \text { Glu }}$

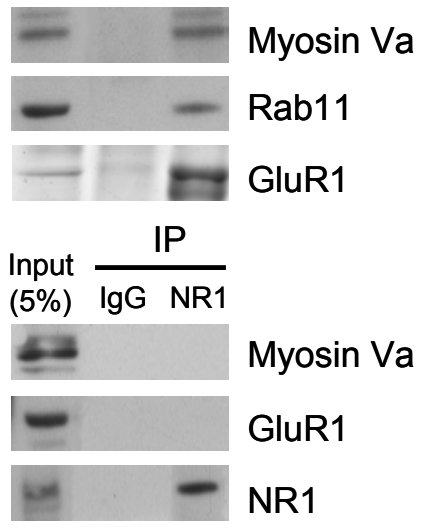

Hippoc. neurons

Input GST- GST-

(5\%) GST M-tail G-tail

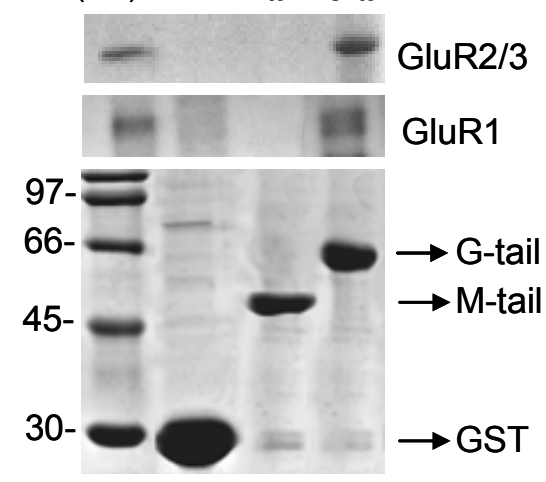

COS-7 cells

Input
$(5 \%)$ GST $\quad \begin{aligned} & \text { GST- } \\ & \text { G-tail }\end{aligned}$

- $\quad$ EGFP-Rab11

myc-GluR1

myc-GluR1- $\Delta 30$

$66-$

$30-$

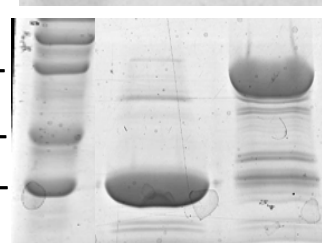

$\rightarrow$ G-tail
His-tag-purified

Input GST-

(5\%) GST G-tail

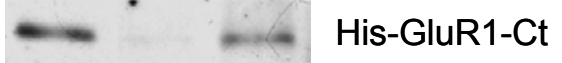

66- $\rightarrow$ G-tail

$45-$

$30-$

$\rightarrow$ GST

\section{Figure 1}




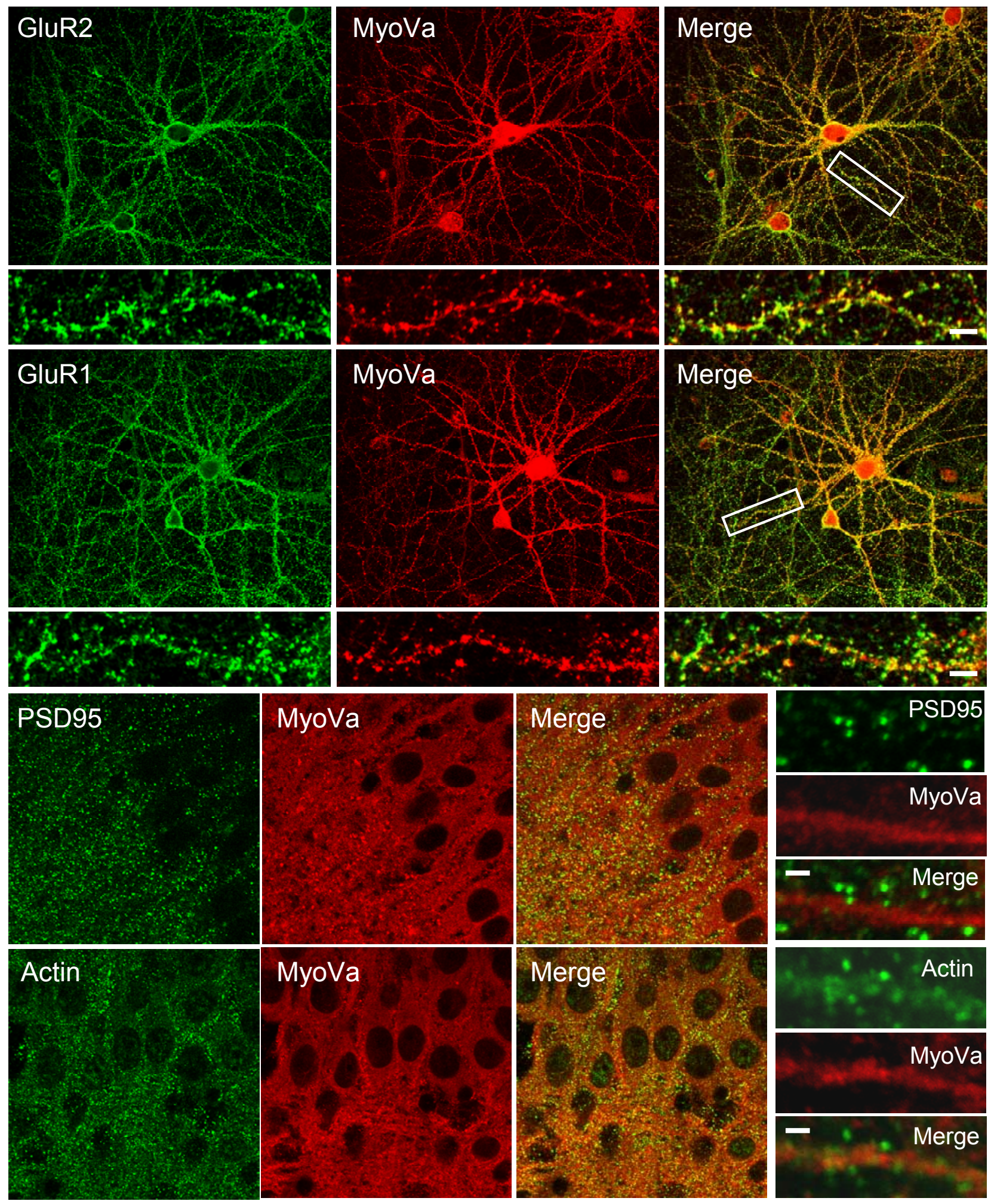

Figure 2 

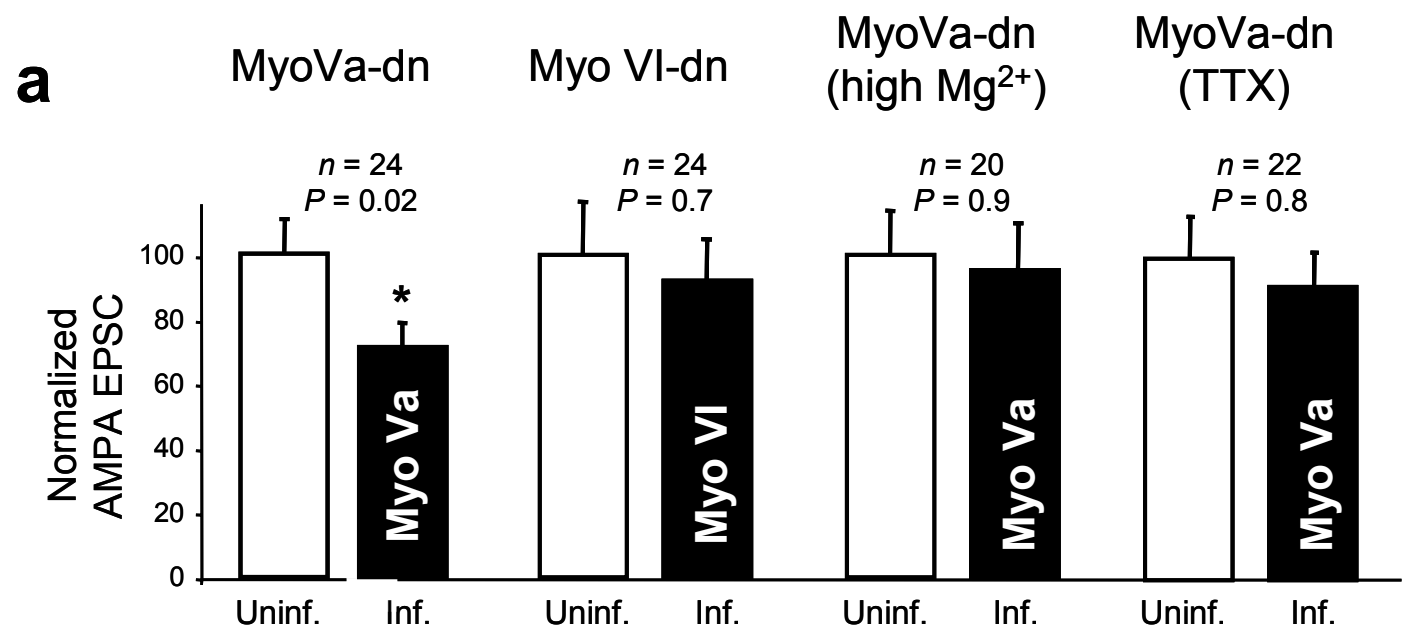

b
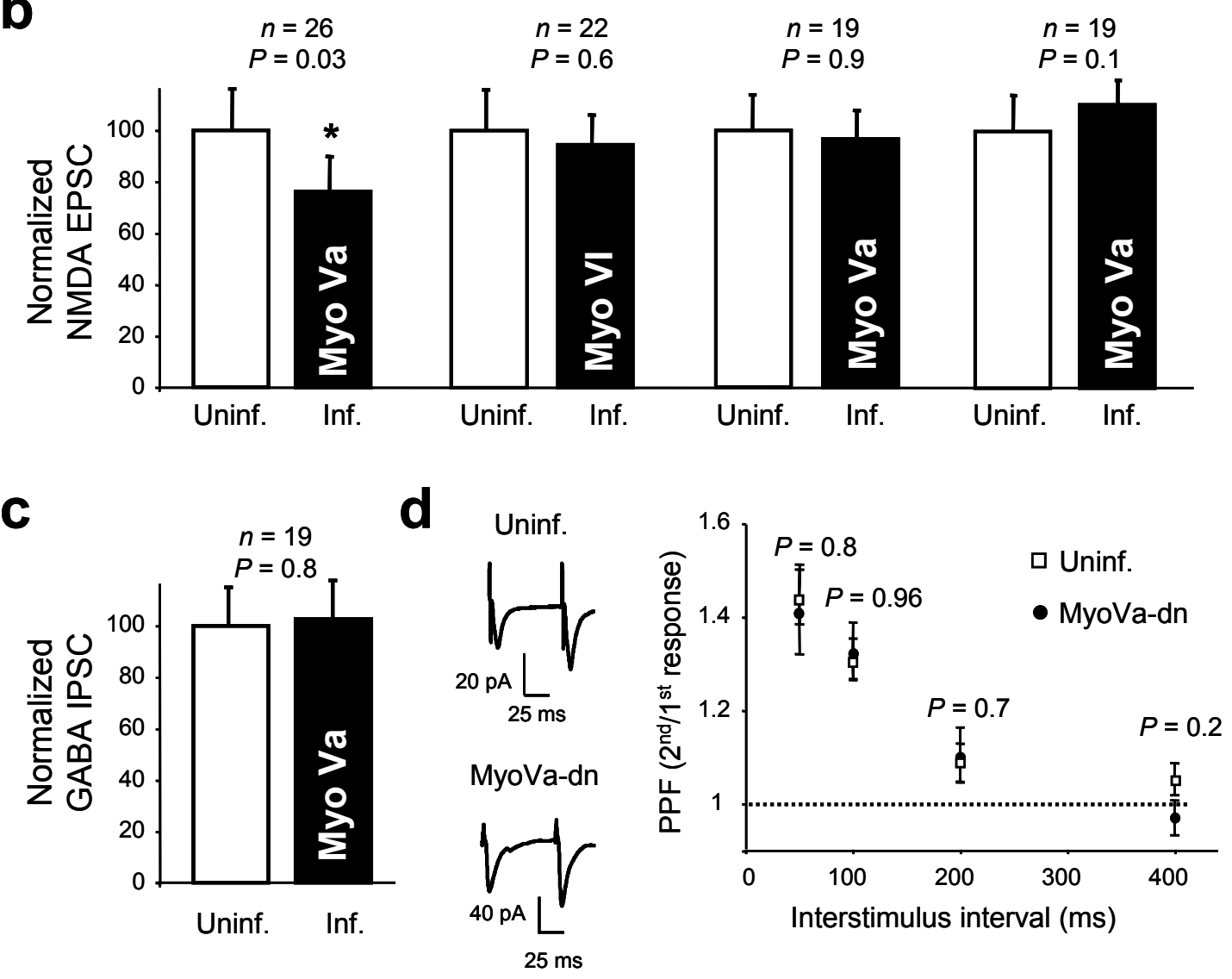

\section{Figure 3}




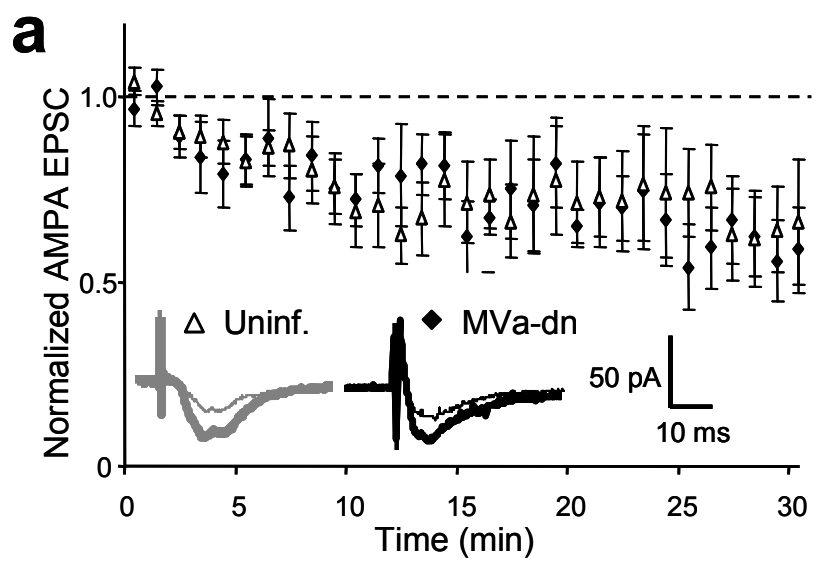

b

C $\diamond$ Untr. $\square$ MVa-dn $\square$ siRNA d
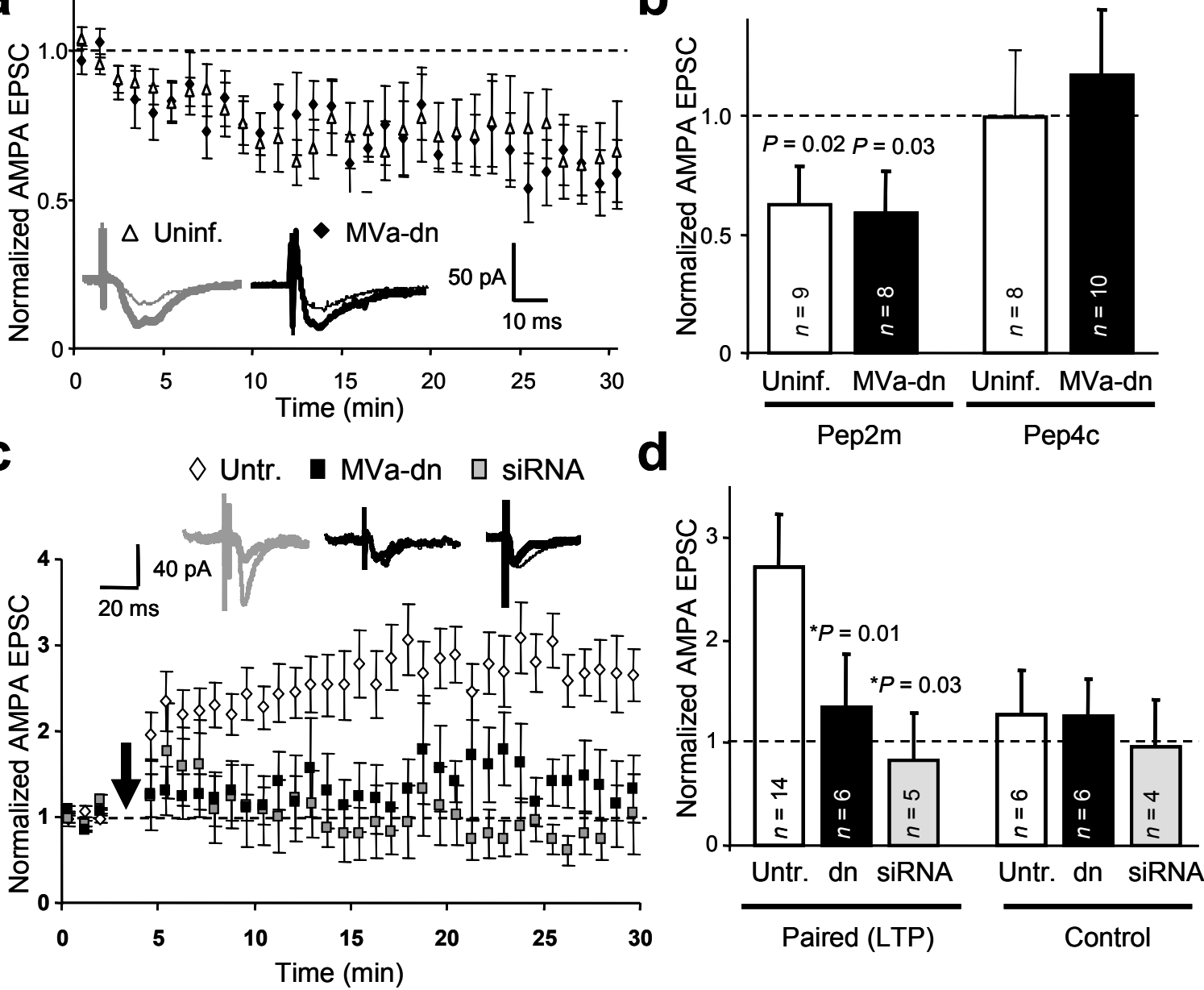

e
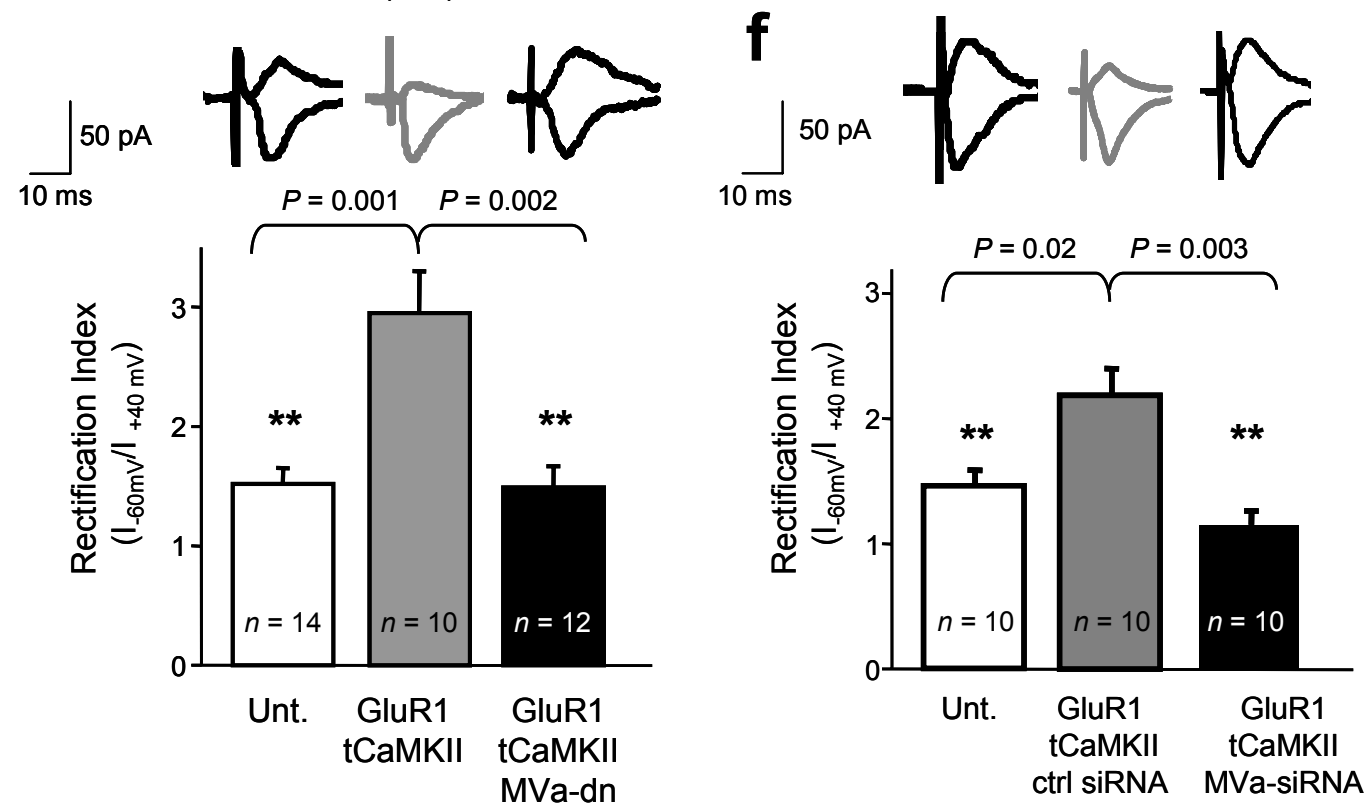

Figure 4 
a

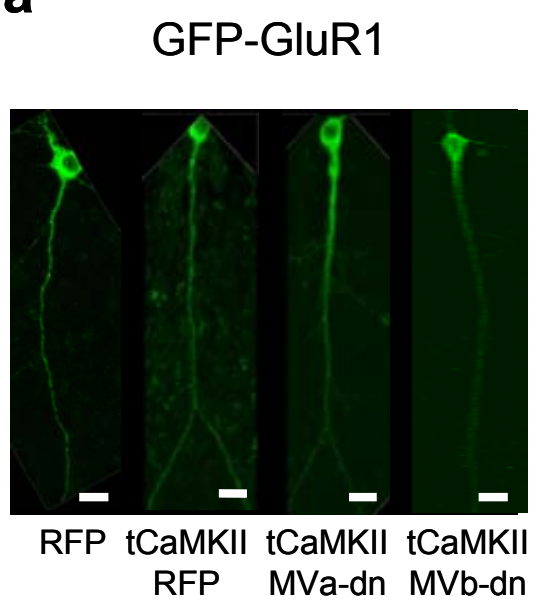

d

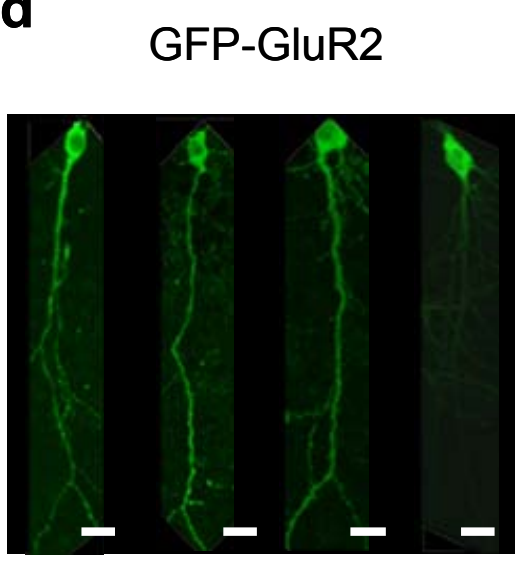

RFP MVa-dn MVb-dn Vinc. b

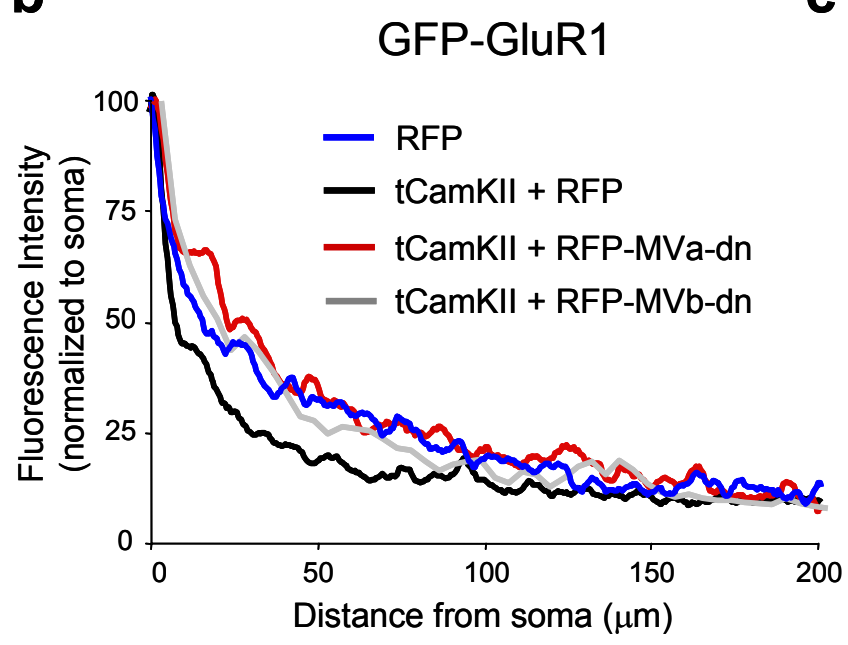

e

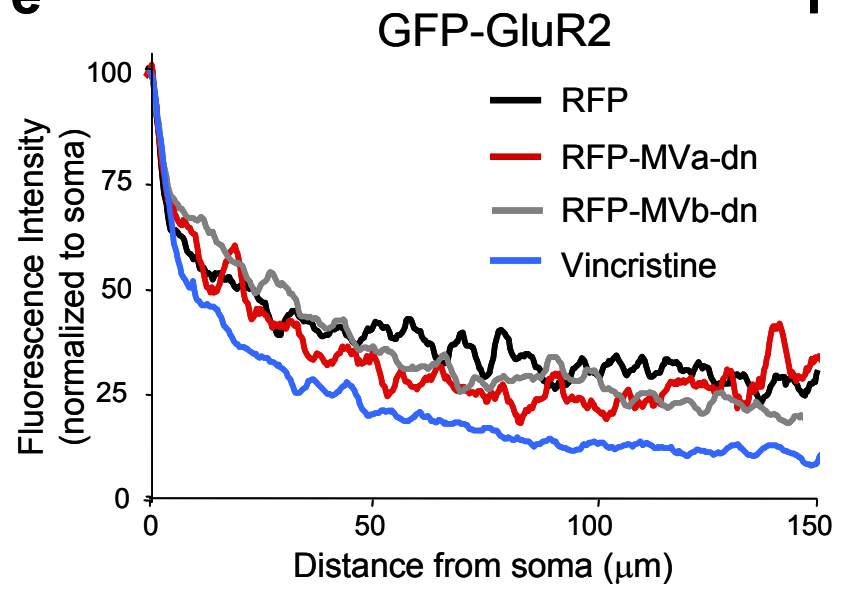

C

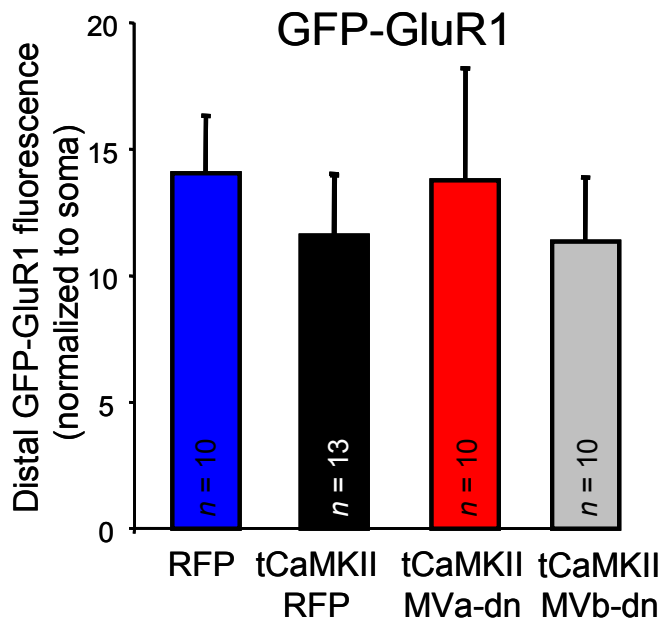

f

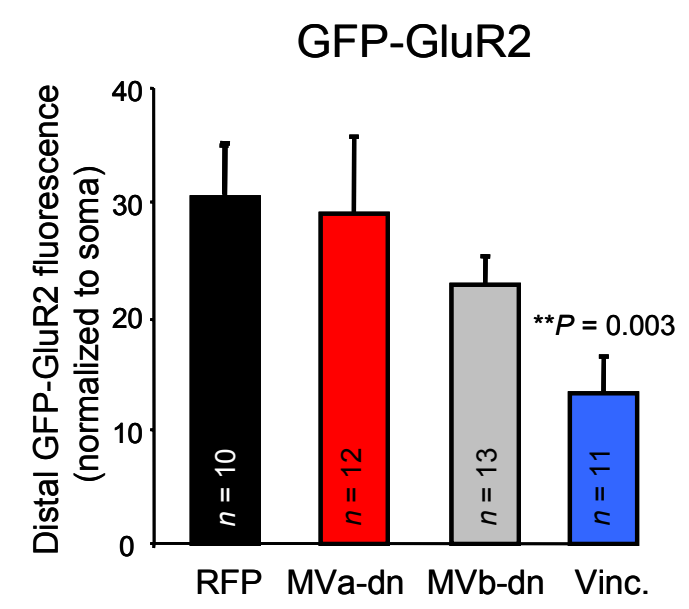

Figure 5 
a

GFP-GluR1 + tCaMKII

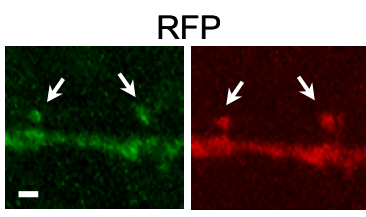

GFP-GluR1 + tCaMKII RFP-MVa-dn

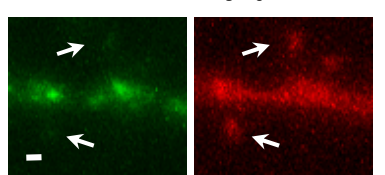

d

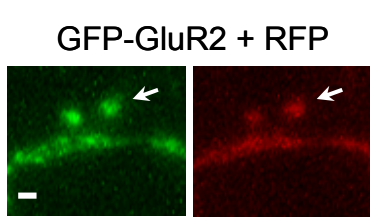

GFP-GluR2

RFP-MVa-dn

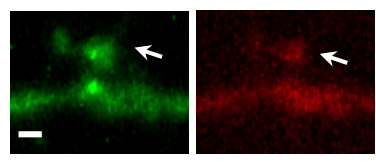

b

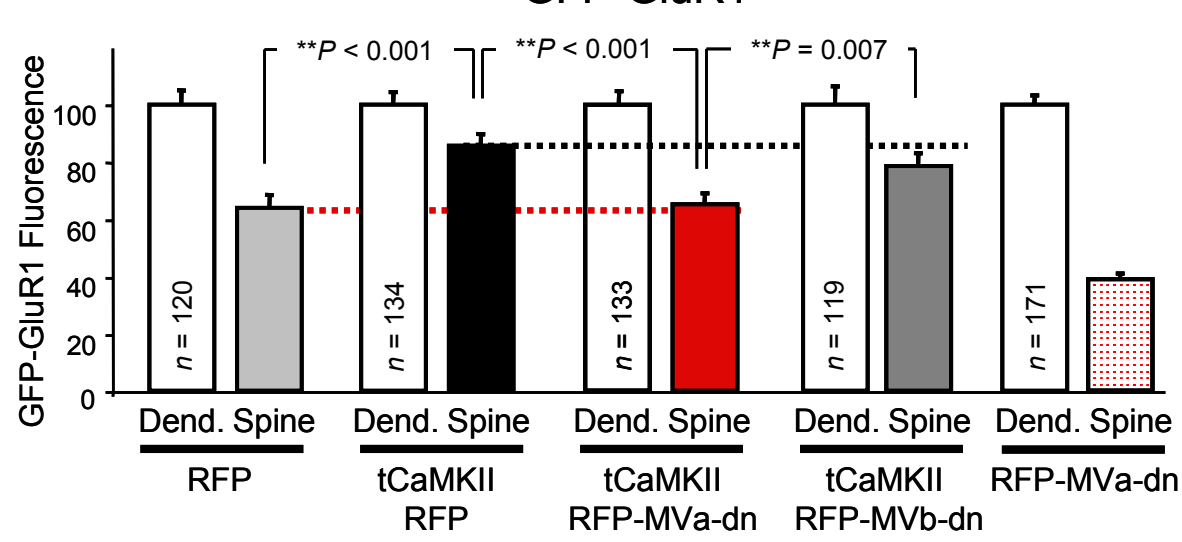

e

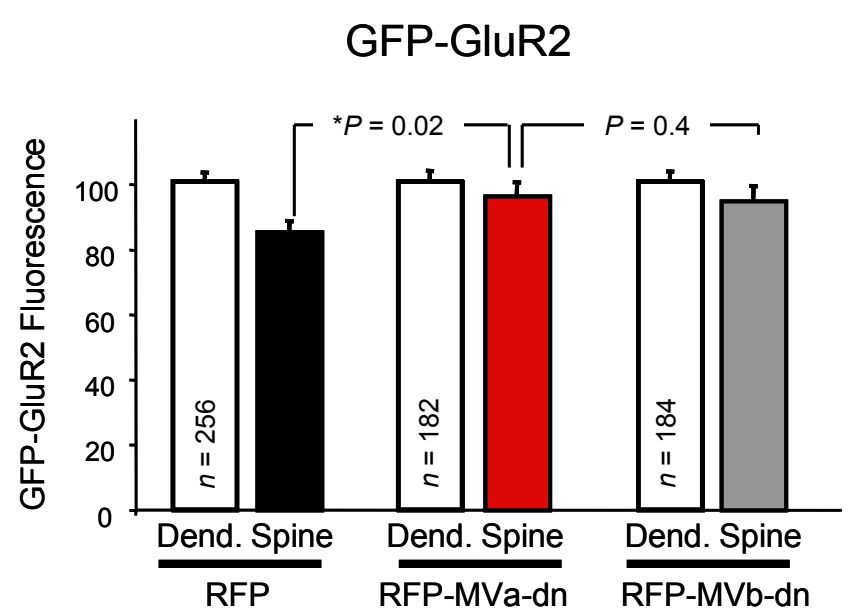

c

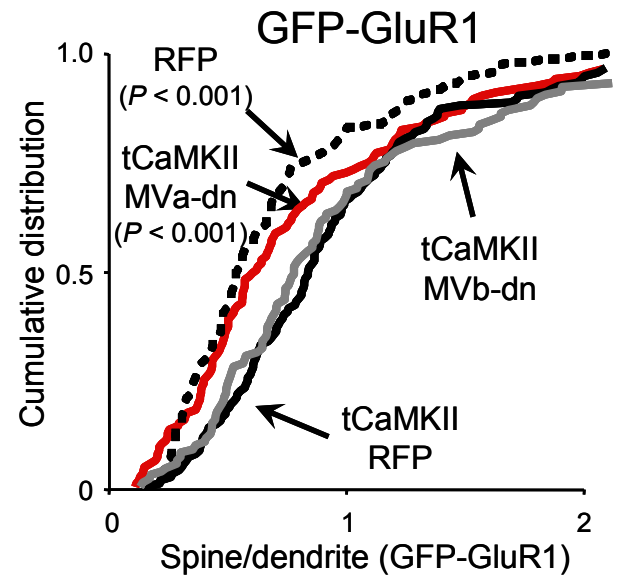

f

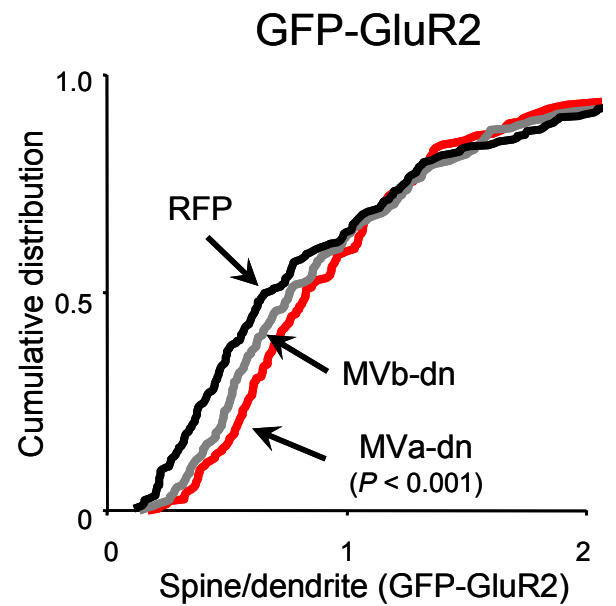

Figure 6 
a

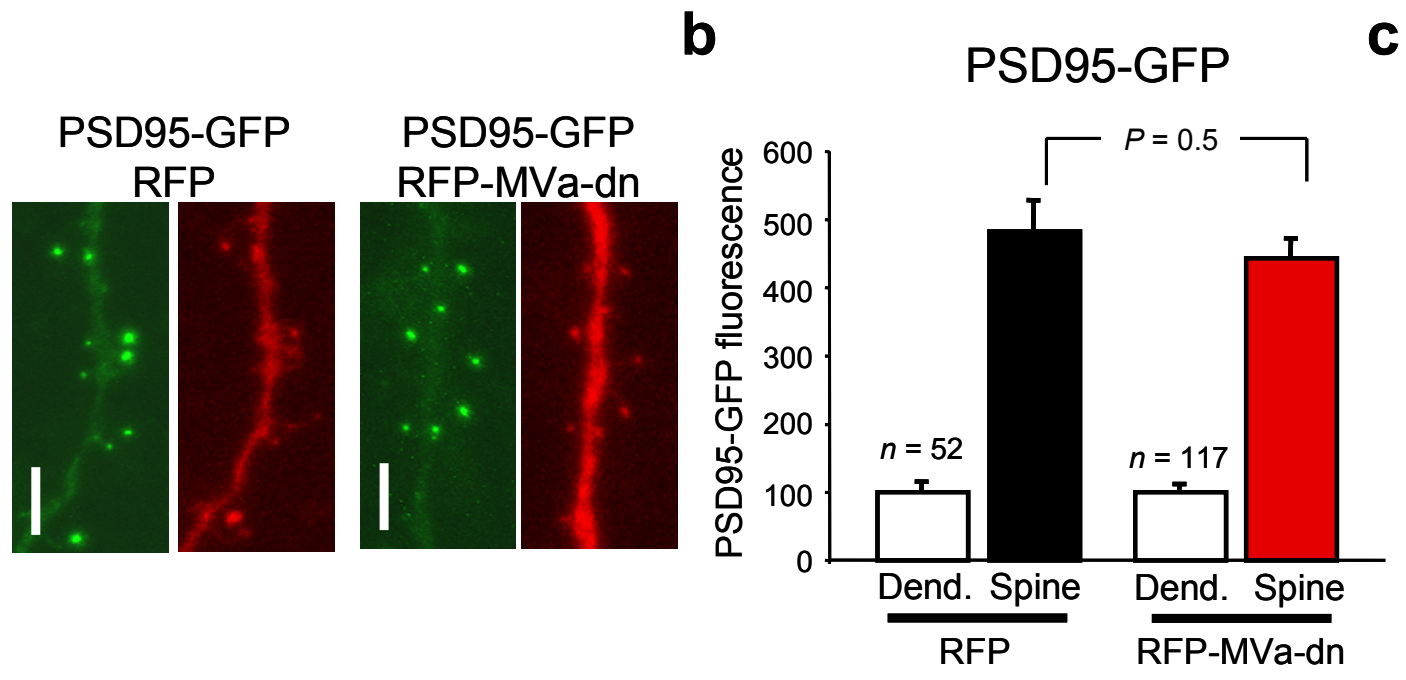

d

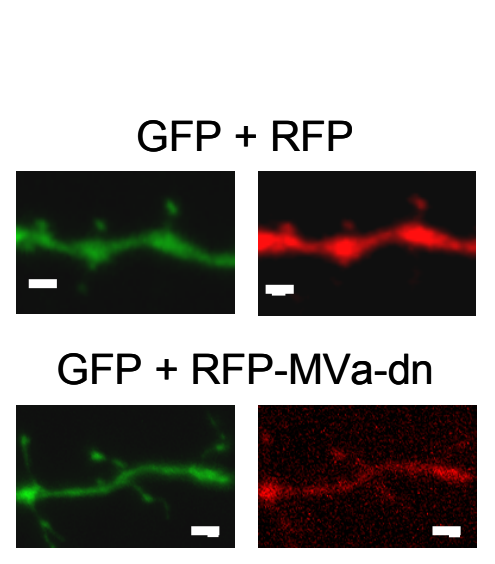

e

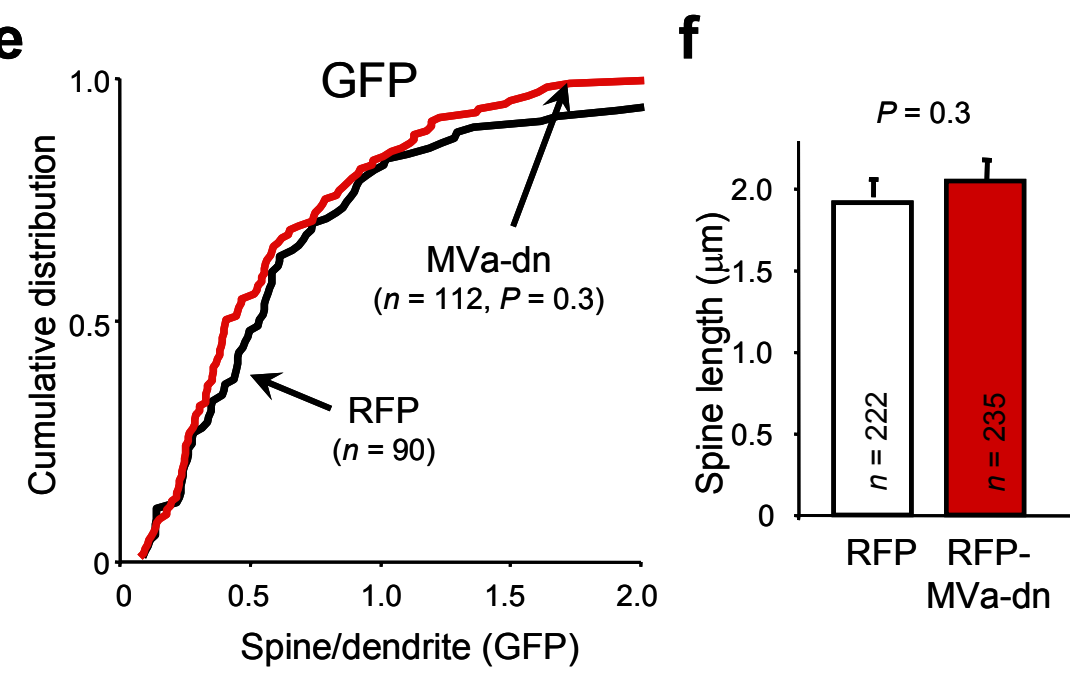

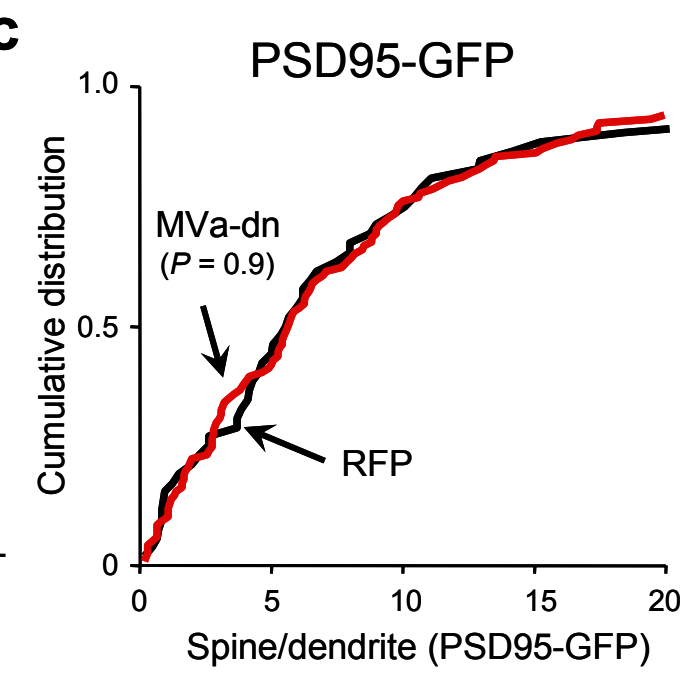

Figure 7 
a

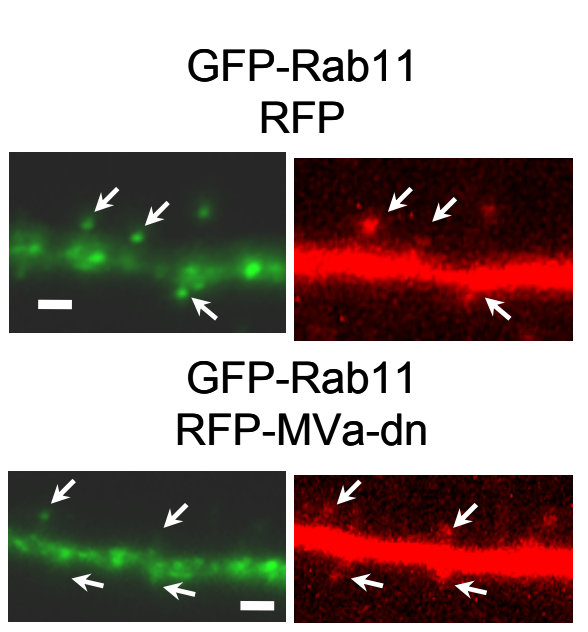

b

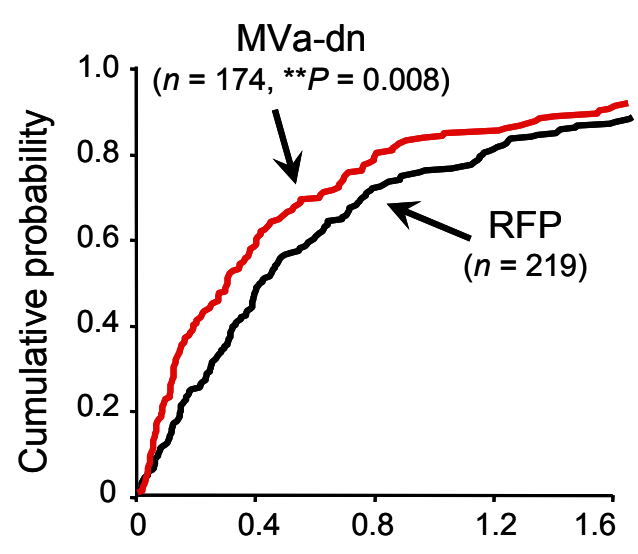

Spine/dendrite (GFP-Rab11)

C

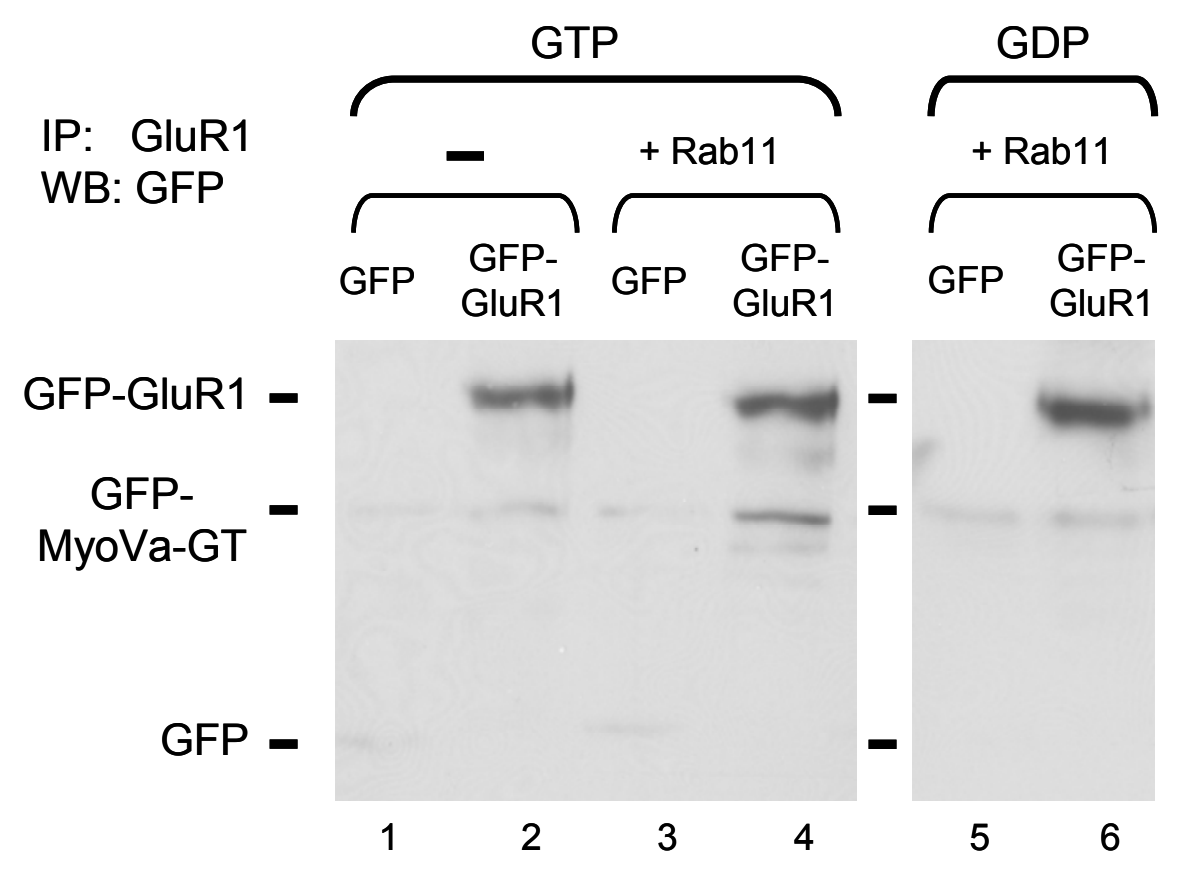

Figure 8 


\section{SUPPLEMENTARY INFORMATION}

\section{METHODS}

Fluorescence imaging

Primary hippocampal neurons (DIV21) or organotypic hippocampal slices were fixed with $4 \%$ paraformaldehyde plus $4 \%$ sucrose and processed for imunostaining. Primary antibodies were rabbit anti-Myosin Va (Sigma), and mouse anti-GluR1 (Epitomics), anti-GluR2 (Chemicon), anti-PSD95 (Upstate) and anti-actin (Chemicon). Secondary antibodies were anti-rabbit conjugated to Alexa 594 and anti-mouse conjugated to Alexa 488. Images were obtained with an Olympus FV500 confocal microscope with a $60 x$ oil immersion lens. Digital images were acquired using the FluoView software and were reconstructed and analyzed using Image $\mathrm{J}$ software. Dendritic and spine distributions of GFP-tagged proteins was quantified as previously described ${ }^{36}$.

Preparation of hippocampal extracts and Western blot analysis

Hippocampal slices from organotypic cultures were homogenized in $10 \mathrm{mM} H E P E S, p H$ 7.4, $500 \mathrm{mM} \mathrm{NaCl}, 10 \mathrm{mM} \mathrm{NaF}, 1 \mu \mathrm{M}$ Microcystin LR, $0.5 \mu \mathrm{M}$ Calyculin A, $10 \mathrm{mM}$ EDTA, $0.1 \mathrm{mM}$ PMSF, $2 \mu \mathrm{g} / \mathrm{ml}$ CLAP (cocktail of Chymostatin, Leupeptin, Pepstatin A and Antipain) and $1 \%$ Triton $\mathrm{X}-100$. Insoluble fraction was discarded and soluble fraction samples were denatured and resolved by SDS-PAGE. Primary antibodies for Western blot analysis were from Chemicon (GluR1, GluR1 phospho-S831, GluR2/3, NR2B, 
$\alpha$ CamKII phospho-T286 and actin), Sigma (Myosin Va), Upstate (PSD95 and $\alpha$ CamKII), BD Biosciences (Rab8 and Rab11), Santa Cruz (GKAP) and Roche (GFP).

\section{Supplementary Figure 1. Myosin Vb-dn does not affect AMPAR-mediated synaptic} transmission. Organotypic slice cultures were infected with GFP-tagged Myosin Vb-dn, Double whole-cell recordings were established from pairs of uninfected and infected CA1 neurons under voltage-clamp configuration. The amplitude of the evoked postsynaptic response mediated by AMPA or NMDA receptors was normalized to the uninfected neurons and plotted as averages \pm standard error of the mean. " $n$ " represents number of cell pairs. To note, Myosin Vb-dn produced a significant depression of NMDAR-mediated currents, which we have not explored further.

\section{Supplementary Figure 2. Myosin Va-dn does not affect expression of several synaptic proteins in CA1 hippocampal neurons. Extracts of CA1 regions from organotypic hippocampal slices uninfected or infected with GFP-Myosin Va-dn were resolved by SDS-PAGE and analyzed by Western blotting. A. Average immunoreactivity of each indicated protein from infected slices was normalized to the control (uninfected) condition and plotted as a percentage \pm standard error of the mean ( $n>6$ for each protein). Values for phosphorylated proteins (GluR1 P-S831 and $\alpha$ CaMKII P-T286) were divided by the total levels of the corresponding protein $\mathbf{B}$. Representative western blots for the data shown in A.}




\section{Supplementary Figure 3. Characterization of a specific siRNA against Myosin Va.}

A. COS-7 cells were transfected with GFP-tagged Myosin Va globular tail (aa 10051830) together with an anti-Myosin Va small interference RNA (MyoVa siRNA), a scrambled siRNA or pSuper empty vector (see Methods for specific sequences). Cell extracts were prepared 24 hours after transfection and were analyzed by western blot using antibodies against GFP (top panel) or actin (bottom panel). Histogram: Quantification of Myosin Va globular tail expression from three independent experiments as the one shown above. MyoVa siRNA produced a significant suppression of Myosin Va as compared to the empty vector $(p=0.04)$ and the scramble siRNA ( $p=0.01$ ) B. Hippocampal neurons (DIV 3) were transfected with GFP and either with anti-MyoVa siRNA or scrambled siRNA. Four days after transfection, neurons were fixed and stained for endogenous Myosin Va. Reduction in Myosin Va staining is apparent in neurons transfected with anti-MyoVa siRNA (right panels; arrowhead) but not with the scramble siRNA (left panels; arrowhead). Scale bar, $10 \mu \mathrm{m}$. C. Quantification of endogenous Myosin Va expression from experiments as the one shown in C, after 2 days (left) or 4 days (right) of siRNA expression. Levels of Myosin Va staining intensity were normalized to untransfected neurons in the same field. AntiMyoVa siRNA significantly reduced the expression levels of endogenous Myosin $\mathrm{Va}$ after both 2 and 4 days ( $p=0.002$ and $p<0.0001$, respectively).

Supplementary Figure 4. Myosin Va is required for PSD95-driven synaptic delivery of GluR1. AMPAR mediated responses were recorded at $-60 \mathrm{mV}$ and +40 $\mathrm{mV}$. The rectification index $(\mathrm{RI})$ was calculated as the ratio of responses at these 
holding potentials. RI was determined for untransfected CA1 neurons and for neurons transfected with GFP-GluR1 plus PSD95 (A), or GFP-GluR1 plus PSD95 and GFPtagged Myosin Va-dn (B). "n" represents number of cells. Statistical significance was calculated with Mann-Whitney test. Sample traces are shown above the corresponding columns of the plot.

Supplementary Figure 5. Schematic model for the role of Myosin Va and Rab11 in the regulated delivery of AMPA receptors into spines. Left part ("constitutive") depicts the continuous (activity-independent) delivery of GluR2/GluR3 receptors, which does not require Myosin Va. Right part ("LTP”) represents the activity-dependent translocation of GluR1/GluR2 receptors mediated by Myosin Va and Rab11. In this case, receptor transport may be triggered by Myosin Va activation upon NMDAR activation and $\mathrm{Ca}^{2+}$ entry, or by specific modifications in the GluR1 subunit that enable it to associate with the motor complex (see further explanations in the main text). As recently reported, final synaptic delivery after receptor entry into the spine is thought to be mediated by a different endosomal compartment, controlled by Rab $8^{41}$. Myosin Va motor and cargo binding domains are represented in pink and blue, respectively. 


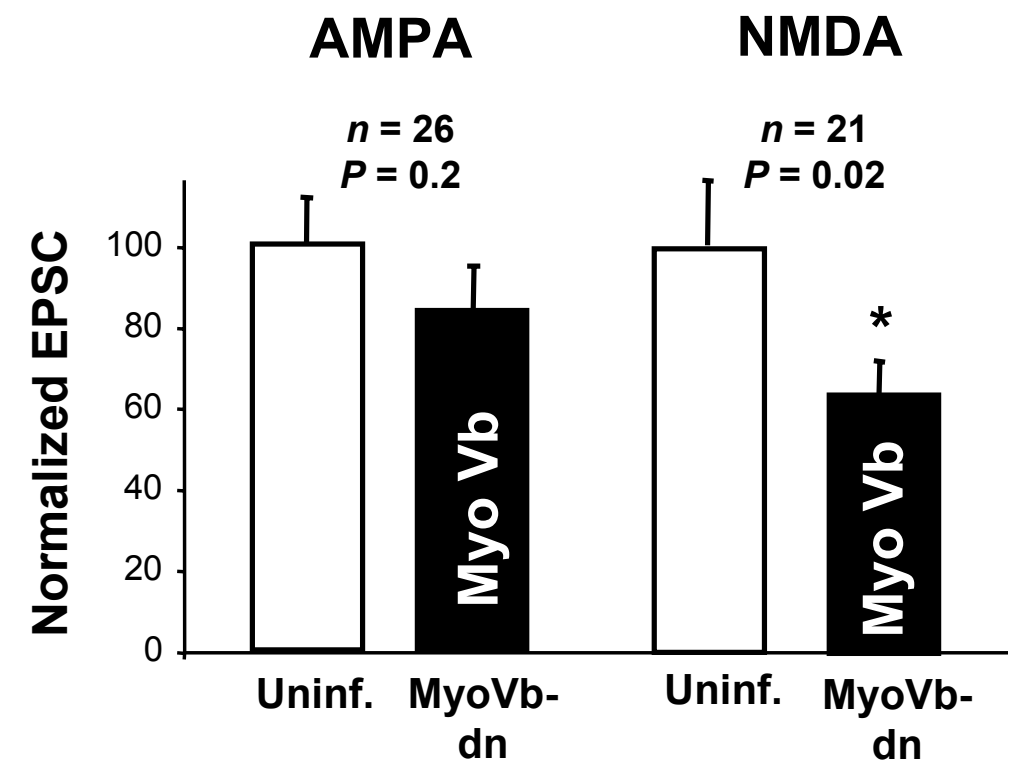

Supplementary Figure 1 
A

B
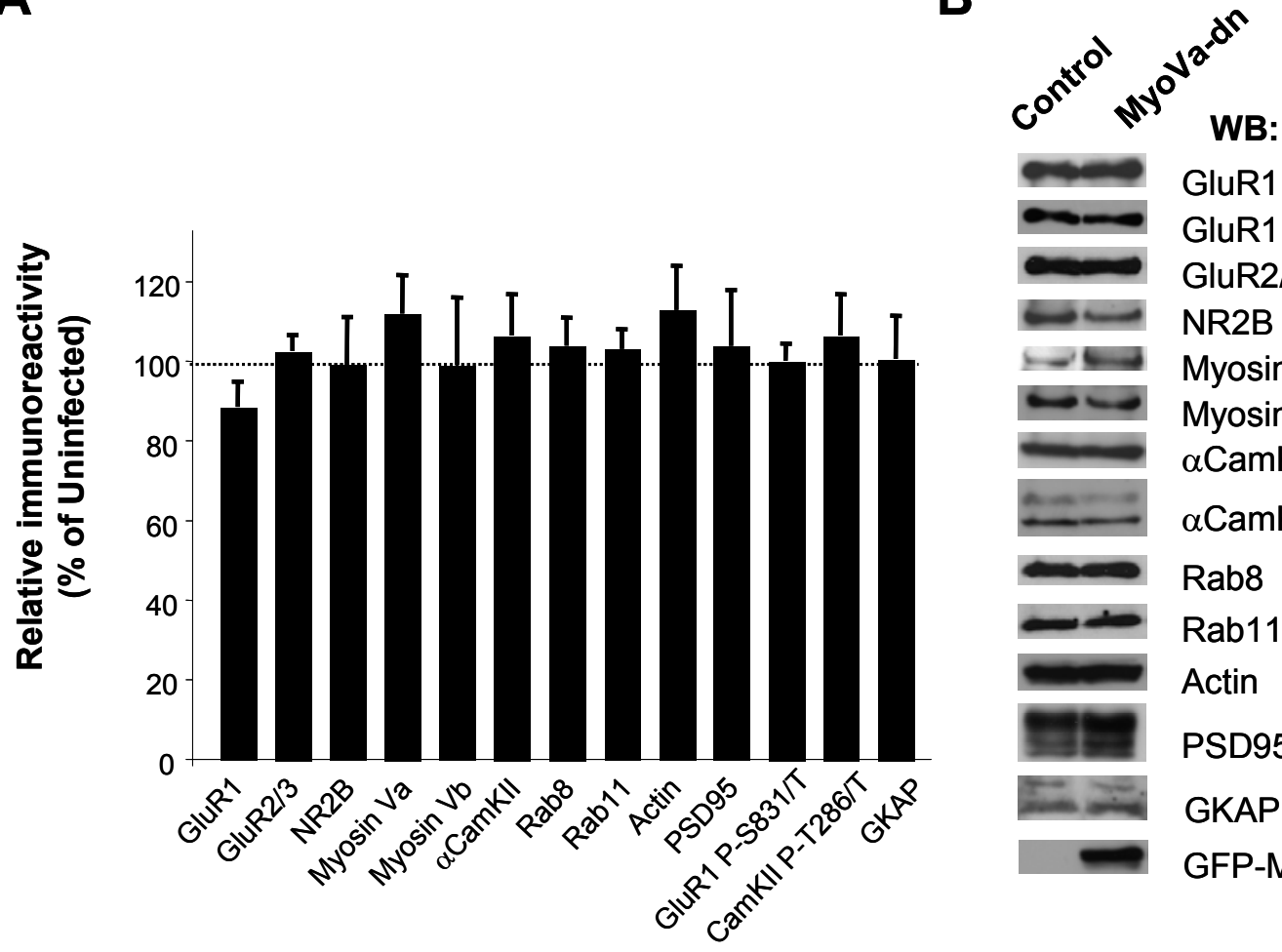

GluR1

GluR1 P-S831

GluR2/3

NR2B

$\longrightarrow$ Myosin Va

Myosin Vb

$\alpha$ CamKII

$\alpha$ CamKII P-T286

Rab8

Rab11

Actin

PSD95

GKAP

GFP-Myosin Va-dn

Supplementary Figure 2 
a
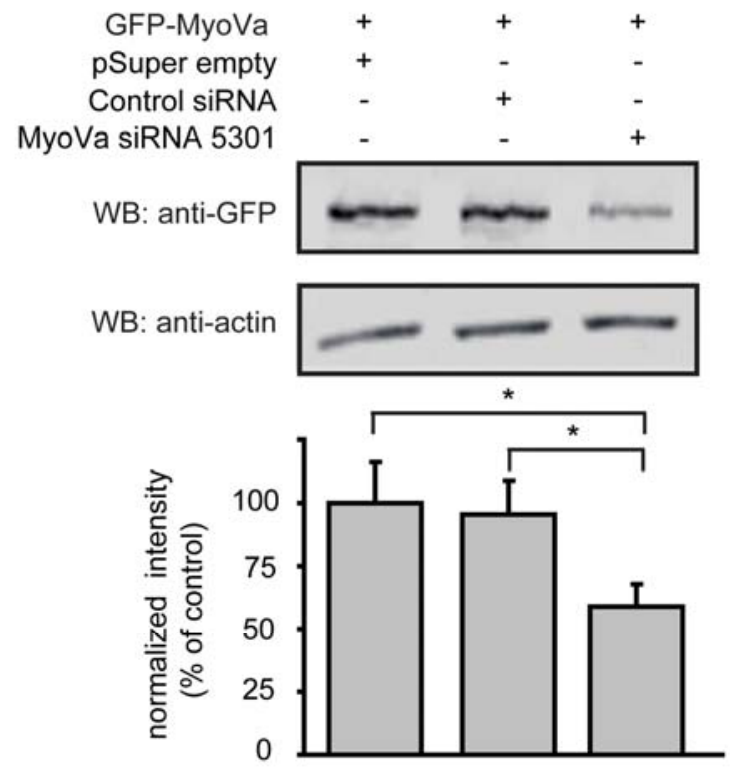

b
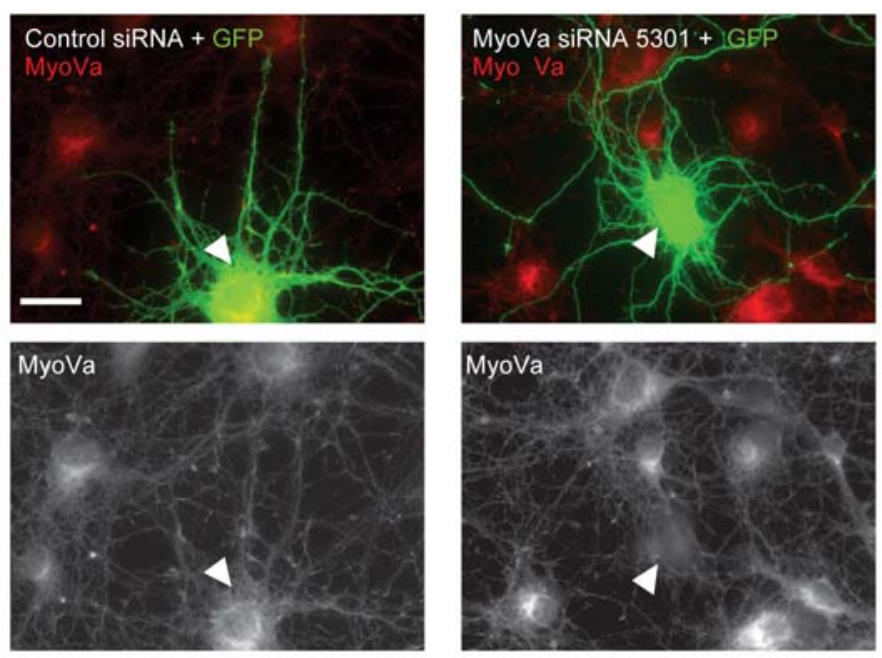

C

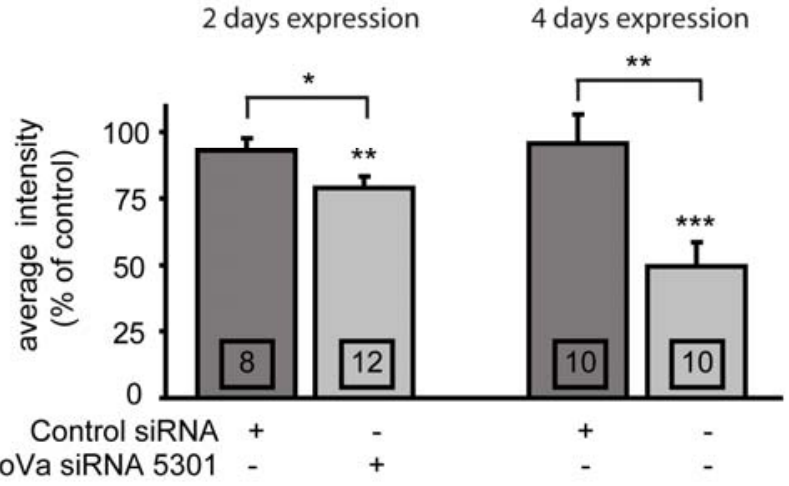

Supplementary Figure 3 
A

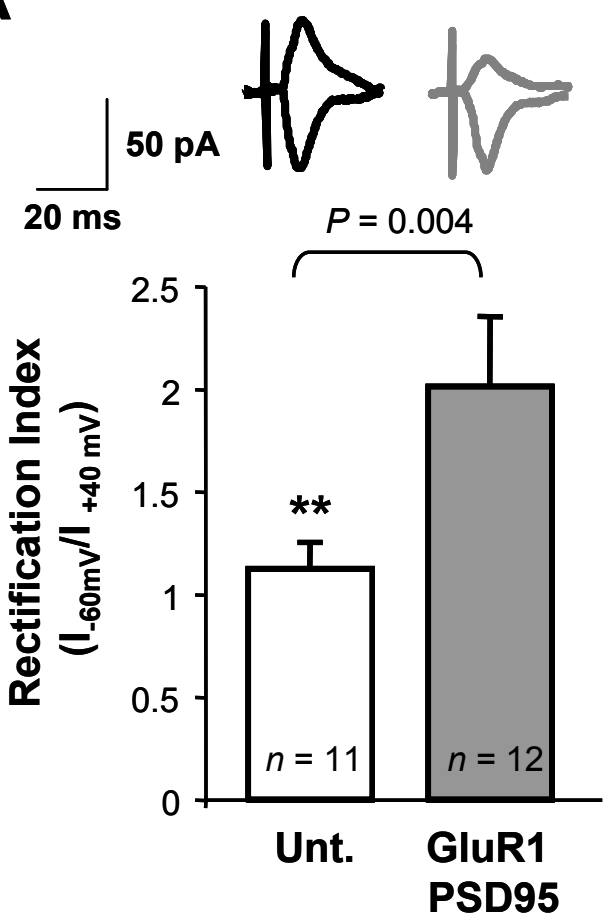

B

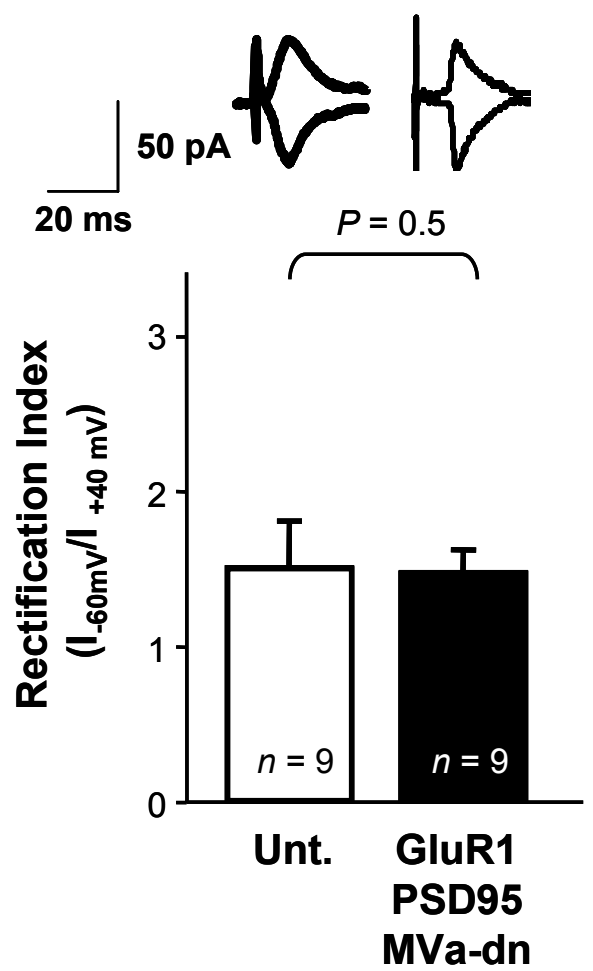

Supplementary Figure 4 


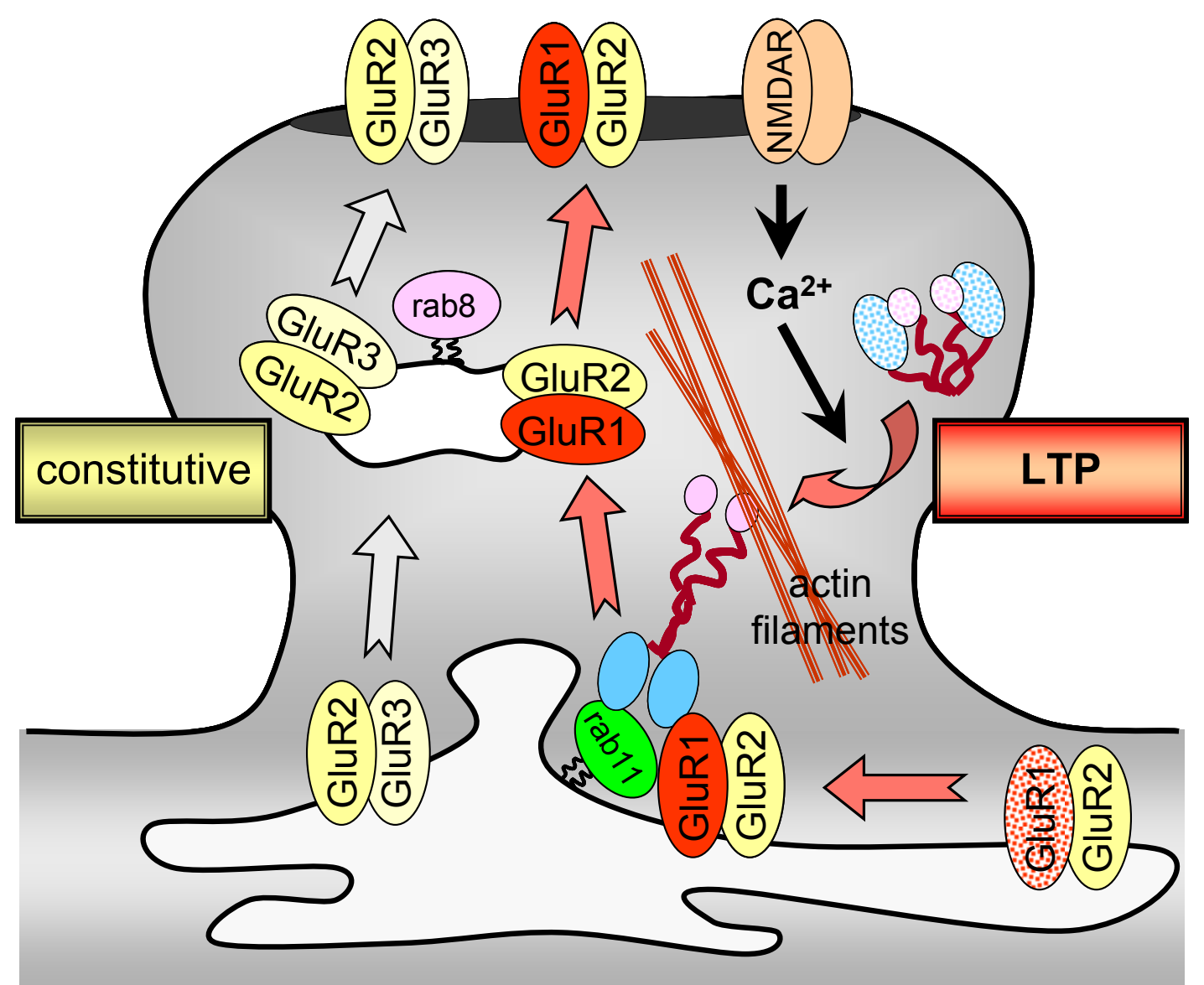

inactive
Myosin Va
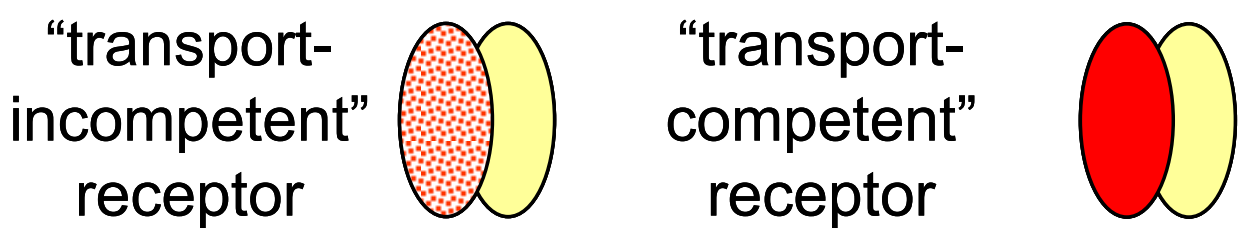

Supplementary Figure 5 